\title{
transgenerational impact: a prospective study on small regulatory microRNAs
}

Received

19 June 2014

Accepted

24 November 2014

Published

17 December 2014

Correspondence and requests for materials should be addressed to B.Z. (zhangb@ecu. edu)
Faten A. Taki ', Xiaoping Pan' ${ }^{1}$, Myon-Hee Lee ${ }^{2}$ \& Baohong Zhang'

'Department of Biology, East Carolina University, Greenville, NC 27858, USA, ${ }^{2}$ Department of Medicine, Brody School of Medicine, East Carolina University, Greenville, NC 27834, USA.

Early developmental stages are highly sensitive to stress and it has been reported that pre-conditioning with tobacco smoking during adolescence predisposes those youngsters to become smokers as adults. However, the molecular mechanisms of nicotine-induced transgenerational consequences are unknown. In this study, we genome-widely investigated the impact of nicotine exposure on small regulatory microRNAs (miRNAs) and its implication on health disorders at a transgenerational aspect. Our results demonstrate that nicotine exposure, even at the low dose, affected the global expression profiles of miRNAs not only in the treated worms (F0 parent generation) but also in two subsequent generations (F1 and F2, children and grandchildren). Some miRNAs were commonly affected by nicotine across two or more generations while others were specific to one. The general miRNA patterns followed a "two-hit" model as a function of nicotine exposure and abstinence. Target prediction and pathway enrichment analyses showed daf-4, daf- 1 , fos-1, cmk-1, and unc-30 to be potential effectors of nicotine addiction. These genes are involved in physiological states and phenotypes that paralleled previously published nicotine induced behavior. Our study offered new insights and further awareness on the transgenerational effects of nicotine exposed during the vulnerable post-embryonic stages, and identified new biomarkers for nicotine addiction.

$40 \%$ of children have been estimated to be exposed to nicotine and up to $60 \%$ of teenagers were reported to be active smokers in some developing countries in $2010^{1,2}$. Whether actively or passively taken, tobacco smoke causes damage to every organ in the body. High death rates and increased economic burden have triggered a lot of policies and research on tobacco-related diseases. Despite its known health risks, tobacco abusers are entrapped in a vicious cycle of drug dependence. Among the 4000 chemicals in tobacco, nicotine is one of the primarily addictive components mediating continuous smoking relapses throughout an individual's lifetime $e^{3-5}$. Early developmental periods are known to be highly sensitive and vulnerable to stress. Children who are exposed to smoke are more susceptible to become smokers during adulthood ${ }^{6,7}$. Numerous studies have reported the effects of nicotine ${ }^{8-15}$ which focused on the exposed generation. Other studies investigated the effects of nicotine during prenatal and perinatal periods ${ }^{16-19}$. However, to our knowledge, the transgenerational effect of nicotine exposed strictly till adolescence has not been reported. Our recent studies show that nicotine exposure caused significant behavioral changes in C. elegans ${ }^{20}$. However, the molecular mechanisms of nicotine-induced transgenerational consequences are not known. Therefore, elucidating the molecular regulatory mechanism for such phenotypes is essential for developing new approaches for monitoring and treating nicotine-related health problems.

Research on nicotine was based on many biological organisms (rats, mice, flies, fishes, and worms) ${ }^{21}$ that modeled different aspects of nicotine-induced behavior like sensitization, tolerance, withdrawal, and reinforcement. We employed the nematode C. elegans to investigate the mechanism of action of nicotine on multiple levels. Research on C. elegans is relatively inexpensive, practical, and free of ethical concern. C. elegans is particularly attractive for transgenerational studies due to its small size ( $1 \mathrm{~mm}$ adult), short generation time (2-3 days) and lifespan (2-3 weeks) at $20^{\circ} \mathrm{C}$, and superfluous offspring production (300 eggs/ $\mathrm{N} 2$ hermaphrodite ${ }^{22}$. C. elegans shares up to $80 \%$ homology with the human genome ${ }^{23,24}$. Thus, it has provided a wealth of information about biological and physiological processes over the last decade, the most recent of which involved the discovery of the microRNAs (miRNAs) ${ }^{25}$.

Serious research has been devoted to dissect the factors involved in gene regulation and has provided clues concerned with the environmental contribution in shaping physiological phenotypes. miRNAs are an extensive 
class of newly discovered small regulatory RNAs ${ }^{26}$. Over 200 and 1000 miRNAs have been sequenced in C. elegans and humans, respectively. Due to their conserved and pleiotropic roles in gene regulation processes ${ }^{27-31}$, miRNAs are considered biomarkers of an innate response to environmental fluctuations. Nicotine altered miRNA expression levels in different biological systems (e.g. PDLSC, mouse fetal neuroepithelial precursors, rodents and PC12 cell model, canines, humans) $)^{32-37}$. Some were linked to disruptions in stem cell regeneration ${ }^{32}$. Others were involved in tumorigenesis (e.g. let-7, miR-16 and miR-21) $)^{37,38}$. Nicotine also antagonized and upregulated ethanol-induced miRNAs ${ }^{33}$. Interestingly, a study demonstrated the role of miR-140* in nicotine addiction using rodents and PC12 cells ${ }^{36}$. The research showed that miR-140* targeted dynamin, which is crucial for neuronal plasticity and hence addiction-related processes $^{36}$. Taken collectively, these studies showed a role or miRNAs in nicotine-dependent mechanisms. In this study, we systematically investigated the transgenerational impact of nicotine on miRNA expression and its implication on nicotine-induced health disorders. We took advantage on the wealth of data available on nicotine dose response curves. The latter are specific to each model organism and experimental design, yet are phenotypically comparable. Thus, we chose two nicotine doses associated with a stimulatory versus depressive effects in our C. elegans model as reported by previous studies $^{12,20}$.

\section{Methods}

Nicotine exposure and sampling. Nicotine exposure and worm sampling were based on our previous method ${ }^{20}$. Briefly, nicotine (Acros Organics, NJ, USA) was dissolved in phosphate buffer as $1 \mathrm{M}$ and $0.001 \mathrm{M}$ stocks. $\mathrm{NaCl}$, peptone, agar and water mixture were first autoclaved, cooled and then kept at $55^{\circ} \mathrm{C}$. After the addition of cholesterol, $\mathrm{CaCl}_{2}, \mathrm{MgSO}_{4}$ and $\mathrm{KH}_{2} \mathrm{PO}_{4}$, the medium was divided into three flasks. The first flask was dedicated for control plates (no nicotine) and was left as is. Conversely, an equal amount of nicotine solution was added from each stock to the respective flask to give final concentrations of $20 \mu \mathrm{M}$ and $20 \mathrm{mM}$ in the medium, respectively. Thus, worms were exposed to nicotine uniformly distributed in solid agar medium. The selection of nicotine concentrations $(20 \mu \mathrm{M}$ and $20 \mathrm{mM})$ was based on previous reports ${ }^{12,21}$ followed by a set of assays to confirm the dosedependent biphasic phenotypes. We performed extensive analyses on the effect of nicotine on sinusoidal movement, body bends and reversals, as well as speed ${ }^{20}$. The chosen concentrations were associated with a non-monotonic bell shaped response and thus agreed with previous reports. Thus, $20 \mu \mathrm{M}$ and $20 \mathrm{mM}$ nicotine were considered suitable for our current and intended miRNA study.

C. elegans hermaphrodite N2 Bristol wild type was used. Maintenance and worm transfer were done after NGM plates were seeded with OP50, and then kept at $20^{\circ} \mathrm{C}$. Egg synchronization was done via bleaching $\operatorname{method}^{39}$, with slight modifications. Bleach breaks down the worms allowing for eggs to be free in solution. The eggs were then washed several times and were left to be suspended in the last wash on a shaker in the $20^{\circ} \mathrm{C}$ incubator for about 14 hours. After hatching, all progeny were arrested at L1.

L1 larvae of the parent (F0) generation were transferred to the three treatment groups which included the control group along with the low and high nicotine concentrations. Parent (F0) exposure lasted for about 30 hours until L3/L4 transition. Then, worms were washed off the plates into two eppendorf tubes and pelleted. We controlled for sample bias by collecting all worms on a plate. Thus each biological replicate included worms with variable sensitivities and covered the entire response spectrum within a plate. The bigger pellet was intermittently centrifuged two times at $2000 \mathrm{rpm}$ then $3000 \mathrm{rpm}$ with supernatant removal. Then, it was flash frozen in liquid nitrogen, and stored at $-80^{\circ} \mathrm{C}$ for subsequent molecular studies. The eppendorf with the smaller pellet was washed twice with M9 interrupted by centrifugation and supernatant removal. The worms were then transferred to nicotine-free NGM plates seeded with OP50, left to dry, and were then sealed and placed back in the $20^{\circ} \mathrm{C}$ incubator to grow until second day of adulthood (egg laying peak). Worms were then collected for synchronization of isolated eggs. The whole procedure was repeated twice until F2 generation.

miRNA expression profile. Total RNA was extracted for all treated and control samples using mirVana ${ }^{\mathrm{TM}}$ miRNA Isolation Kit. Briefly, the sample was denatured using a lysis buffer. RNA was then separated from DNA and proteins via acid-phenol extraction. Then, ethanol was added to the sample followed by centrifugation to allow it to pass through a glass-filter. Several washes preceded the elution of the RNA with DNase/RNase-free water. RNA quantification and evaluation was done using the NanoDrop ND-1000 Micro-Volume UV/Vis Spectrophotometer (NanoDrop Technologies, Wilmington, DE).

Reverse transcription was performed using TaqMan microRNA Reverse Transcription kit (Applied Biosystems, Foster City, CA) to reverse transcribe extracted RNA to cDNA for all 231 miRNAs. A total of $200 \mathrm{ng}$ of RNAs was used for each RT reaction. The reactions were then run using thermal cycler for $16^{\circ} \mathrm{C}$ for $30 \mathrm{~min}$ followed by $42^{\circ} \mathrm{C}$ for $30 \mathrm{~min}, 85^{\circ} \mathrm{C}$ for $5 \mathrm{~min}$ and was finally held at $4^{\circ} \mathrm{C}$. The cDNAs were then diluted in $80 \mu \mathrm{L}$ DNase/RNase-free water for qRT-PCR.

The expression levels of miRNAs were analyzed after performing qRT-PCR on 384-well-plate using the ViiA ${ }^{\mathrm{TM}}$ Real-Time PCR System (Applied Biosystem). Briefly, each well carried a $15 \mu \mathrm{L}$ reaction of $5.5 \mu \mathrm{L}$ DNase/RNase free water, 7.5 $\mu \mathrm{L}$ SYBR Green master mix, $1 \mu \mathrm{L}$ diluted cDNA, and $1 \mu \mathrm{L}$ primer mix. A minimum of 3 biological replicates were run. The reaction was run for $10 \mathrm{~min}$ at $95^{\circ} \mathrm{C}$ for enzyme activation followed by denaturation for $15 \mathrm{sec}$ at $95^{\circ} \mathrm{C}$ and an annealing/extension step for $60 \mathrm{sec}$ at $60^{\circ} \mathrm{C}$. The latter 2 steps were repeated for 40 cycles.

The Ct values from the qRT-PCR were exported to an Excel file. The average of the total miRNA (231) Ct-values was used for normalization. The $\Delta \mathrm{Ct}$ values were calculated as $\mathrm{Ct}_{\text {(miRNA) }}-\mathrm{Ct}_{\text {(avg miRNAs) }}$. The $\Delta \Delta \mathrm{Ct}$ was calculated as the difference in the $\Delta \mathrm{Ct}$ values between control and treatment. Then the fold change was calculated as $2^{(\triangle \Delta C T)}$. Statistical analysis was based on t-test for independent samples via SPSS $(20)$ to compare each of the nicotine treatments with control (i.e. low vs. control and high vs. control). Welch test correction was performed to account for unequal variance in each of the compared groups (control and treatment). In addition, to increase the statistical stringency, miRNAs differential expression was deemed significant only if it fulfilled two criteria: $p$ value $<0.05$ and expression changed by at least $50 \%$ relative to control.

Fold change values $\left(2^{(\triangle \Delta C T)}-1\right)$ were used to construct heat maps coupled with non-supervised hierarchical clustering using Euclidean distance and complete linkage analysis and included all miRNAs (vertical axis) and samples (six treatment groups on the horizontal axis). The latter approach was done for miRNAs with statistically significant expression alterations using $\mathrm{MeV}$ (MultiExperiment Viewer $)^{40}$.

Target prediction and pathway analysis. Differentially altered miRNAs that were common to at least two generations were used to perform target prediction using mirSOM software ${ }^{41}$. To prepare the input for analysis, duplicates were removed and thus only unique values of targets with perfect seed match were used. The predicted targets were ranked according to the frequency of occurrence in the originally compiled gene list. Such a frequency reflects the number of miRNAs predicted to target a gene. The list was used as input for DAVID ${ }^{42,43}$ for analysis. Gene ranking was based on functional annotation clustering (highest stringency) endpoint provided by DAVID. Target genes belonging to clusters with enrichment values $\geq 2$ were used based on the order of the clusters to prepare a ranked list. The latter included 321 genes and was used as an input for GOrilla (process ontology) ${ }^{44}$ that provided DAG (directed acyclic graph) showing relationships among enriched processes. miRNAtarget networks were constructed using Cytoscape ${ }^{45}$. Other specific analysis were performed and described in details in their corresponding result section.

\section{Results}

Analyzing the intra-generational effect of nicotine. The effect of nicotine on the parent (F0) generation. We studied the effect of nicotine on the expression levels of 231 miRNAs in L4 C. elegans (N2). The effect of nicotine on the F0 generation can be found in our previous study ${ }^{46}$. To summarize the results, nicotine affected the expression of forty miRNAs (17.3\%), in which three miRNAs (miR-79, miR-80 and miR-230*) were altered in response to the lower nicotine concentration $(20 \mu \mathrm{M})$. The expression of 37 miRNAs was changed after high nicotine concentration $(20 \mathrm{mM})$ treatment. About $78 \%$ of the altered miRNAs were upregulated with fold changes ranging from 0.5 to 3.0 folds. Twenty six miRNAs were upregulated by $>0.5$ folds $(p<0.05$ ). The remaining 22\% miRNAs were downregulated in the $20 \mathrm{mM}$ nicotine treatment group. miR-80 was the only miRNA commonly upregulated in worms treated with low and high nicotine concentrations. Based on the miRNA profile changes observed in response to nicotine in the F0 worm population, we decided to continue our investigations to explore the indirect transgenerational effect of nicotine treatment limited to the postembryonic-stages of the F0 generation.

The effect of nicotine on the offspring (F1) generation. The F0 generation was the only one with direct contact with nicotine, and such was limited to the 30-hour, post-embryonic period (i.e. L1 to L4). With this in mind, we investigated possible effects of nicotine on the genome-wide miRNA expression levels in L4 belonging to the F1 generation. As shown in Figure 1a, the expression of thirty one (13.4\%) unique miRNAs was altered in response to parental nicotine exposure. The low and high nicotine concentrations affected nine and twenty six miRNAs, respectively. Three out of nine miRNAs 

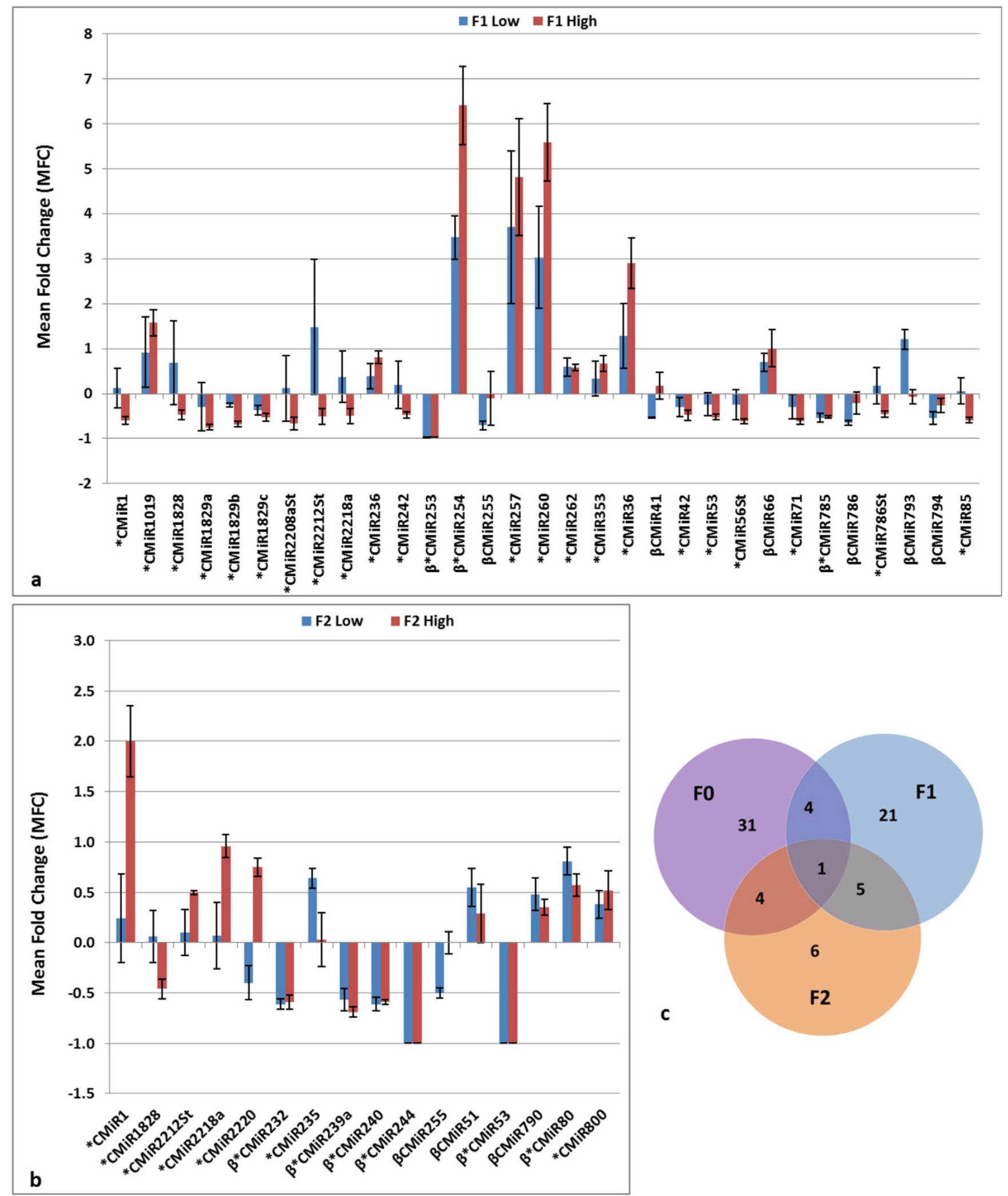

Figure $1 \mid$ Nicotine altered the miRNA expression profiles across generations in a dose-dependent manner. (a) Nicotine significantly altered the expression levels of 31 miRNAs in the F1 worm population. (b) Nicotine significantly altered the expression levels of 16 miRNAs in the F2 worm population. (c) A Venn diagram showing the number of the miRNAs with differentially altered expression levels shared in L4 larvae belonging to the three generations (F0, F1, and F2). P $<0.05$. [ ${ }^{*}, \beta$ denote statistically significant changes in response to high $(20 \mathrm{mM})$ and low $(20 \mu \mathrm{M})$ nicotine concentrations, respectively). All comparisons were based on control. 
were upregulated in offspring of worms exposed to the lower nicotine concentration $(20 \mu \mathrm{M})$ (Table 1$)$. The highest increase was observed for miR-254 (3.5, p = 0.006) followed by miR-793 (1.2, $\mathrm{p}=0.03)$ and miR-66 (0.7, $\mathrm{p}=0.04)$. On the other hand, the remaining six miRNAs were downregulated with the highest fold change observed for miR-253 $(-1.0, \mathrm{p}<0.001)$ followed by miR-255 $(-0.7, \mathrm{p}=$ $0.001)$, miR-786 $(-0.6, \mathrm{p}=0.002)$, and miR-785, miR-794, and miR-41 $(-0.5, p=0.036, p=0.029$, and $\mathrm{p}<0.001$, respectively) (Table 1). As for offspring of parents exposed to the higher nicotine concentration $(20 \mathrm{mM}), 32 \%$ of the differentially altered miRNAs were upregulated. The highest upregulation was observed for miR$254(6.4, \mathrm{p}=0.018)$ followed by miR-260 (5.6, $\mathrm{p}=0.023), \mathrm{miR}-257$ (4.8, $\mathrm{p}=0.034), \operatorname{miR}-36(2.9, \mathrm{p}=0.014), \operatorname{miR}-1019(1.6, \mathrm{p}=0.005)$, miR-236 (0.8, $\mathrm{p}=0.01)$, miR-353 (0.7, $\mathrm{p}=0.016)$, and miR-262 (0.6, $\mathrm{p}=0.014)$. Conversely, $68 \%$ of the miRNAs, altered after parental exposure to high nicotine concentration, were downregulated. With the highest fold change being for miR-253 $(-1.0, \mathrm{p}<0.001)$, others like miR-1829a $(\mathrm{p}=0.006), \operatorname{miR}-1829 \mathrm{~b}(\mathrm{p}=0.001), \operatorname{miR}-2208 \mathrm{a} *(\mathrm{p}$ $=0.041), \operatorname{miR}-56^{*}(\mathrm{p}=0.005), \operatorname{miR}-71(\mathrm{p}=0.011), \operatorname{miR}-1(\mathrm{p}=$ $0.02), \operatorname{miR}-85(\mathrm{p}=0.01), \operatorname{miR}-1829 \mathrm{c}(\mathrm{p}=0.027), \operatorname{miR}-785(\mathrm{p}=$ $0.002), \operatorname{miR}-53(\mathrm{p}=0.001), \operatorname{miR}-2212 \mathrm{St}(\mathrm{p}=0.037), \operatorname{miR}-2218 \mathrm{a}(\mathrm{p}$ $=0.044), \operatorname{miR}-42(\mathrm{p}=0.013), \operatorname{miR}-1828(\mathrm{p}=0.025), \operatorname{miR}-242(\mathrm{p}=$ $0.024)$, and miR-784* $(\mathrm{p}=0.022)$ decreased by more than 0.7 folds (Table 1). Additionally, we observed that three miRNAs were affected by both nicotine concentrations when exposed during the parental post-embryonic stage. miR-254 was the most upregulated in offspring of both treatment groups and as the concentration increased, the magnitude of the upregulation was more dramatic as it doubled from 3.0 folds to 6.0 folds. On the other hand, miR$785(-0.5)$ and miR-253 (-1.0) were downregulated in response to both nicotine concentrations exposed to parents, but unlike miR254 , the magnitude was similar in offspring of parents exposed to both low and high nicotine concentrations (Table 1).

The effect of nicotine on the grand-offspring (F2) generation. We traced down the effect of nicotine until the F2 generation. Similarly, grand-offspring worms were never exposed to nicotine, but progressed from ancestors treated with nicotine during their post-embryonic period. In F2, sixteen unique miRNAs (6.9\%) showed statistically significant alterations in their expression levels in response to grand-parental nicotine exposure (Table 2). Ten and twelve miRNAs were differentially expressed in grand-offsping of parents exposed to low and high nicotine concentrations, respectively. Among the affected miRNAs, $40: 60 \%$ and $50: 50 \%$ were down and upregulated in grand-offspring originating from the parental low and high nicotine treatment groups, respectively (Table 2). Interestingly, six of the sixteen unique miRNAs were commonly and similarly altered in both treatment groups progressing from grand-parents exposed to low and high nicotine concentrations. miR-80 was the only commonly upregulated miRNA by $0.8(\mathrm{p}=$ $0.027)$ and $0.6(\mathrm{p}=0.035)$ fold changes in grand-progenies of parents exposed to low and high doses, respectively. Oppositely, miR$239 \mathrm{a}\left(\mathrm{p}_{(\mathrm{L})}=0.006, \mathrm{p}_{(\mathrm{H})}<0.001\right), \operatorname{miR}-240\left(\mathrm{p}_{(\mathrm{L})}=0.012, \mathrm{p}_{(\mathrm{H})}=\right.$ $0.001)$, and miR-232 $\left(\mathrm{p}_{(\mathrm{L})}<0.001, \mathrm{p}_{(\mathrm{H})}=0.015\right)$ were downregulated in both groups by about 0.6 folds, while miR-53 and miR-244 were downregulated by about 1.0 folds with $\mathrm{p}_{(\mathrm{L})}$ and $\mathrm{p}_{(\mathrm{H})}<0.001$. Other miRNAs were dose-dependent as their expression levels changed in one of the two groups, but not both. For example, miR-235 (p $=0.003)$, miR-51 ( $\mathrm{p}=0.043)$, and $\mathrm{miR}-790(\mathrm{p}=0.041)$ were upregulated by $>0.5$ folds, while miR-255 was downregulated by about

Table 1 | The effect of parental post-embryonic nicotine exposure on miRNA expression levels in L4 worms belonging to the F1 generation. (* and $\beta$ denote $p$-values $<0.05$ in comparison to control)

\begin{tabular}{|c|c|c|c|c|}
\hline & \multicolumn{2}{|c|}{ F1 Low } & \multicolumn{2}{|c|}{ F1 High } \\
\hline & P value & $\mathrm{MFC} \pm \mathrm{SE}$ & $P$ value & $\mathrm{MFC} \pm \mathrm{SE}$ \\
\hline *miR 1019 & 0.321 & $0.92 \pm 0.78$ & 0.005 & $1.58 \pm 0.29$ \\
\hline *miR 1828 & 0.517 & $0.68 \pm 0.93$ & 0.025 & $-0.47 \pm 0.11$ \\
\hline${ }^{*}$ miR 1 829a & 0.637 & $-0.29 \pm 0.53$ & 0.006 & $-0.75 \pm 0.06$ \\
\hline *miR $1829 b$ & 0.009 & $-0.25 \pm 0.04$ & 0.001 & $-0.68 \pm 0.06$ \\
\hline${ }^{*}$ miR221 12St & 0.397 & $1.48 \pm 1.50$ & 0.037 & $-0.51 \pm 0.17$ \\
\hline *miR2218a & 0.581 & $0.37 \pm 0.57$ & 0.044 & $-0.50 \pm 0.17$ \\
\hline *miR236 & 0.313 & $0.38 \pm 0.28$ & 0.010 & $0.81 \pm 0.14$ \\
\hline *miR242 & 0.758 & $0.19 \pm 0.53$ & 0.024 & $-0.47 \pm 0.07$ \\
\hline$\beta * m i R 253$ & 0.000 & $-0.97 \pm 0.01$ & 0.000 & $-0.97 \pm 0.00$ \\
\hline$\beta * m i R 254$ & 0.006 & $3.47 \pm 0.49$ & 0.018 & $6.41 \pm 0.87$ \\
\hline "miR353 & 0.437 & $0.33 \pm 0.39$ & 0.016 & $0.67 \pm 0.17$ \\
\hline "miR36 & 0.175 & $1.28 \pm 0.72$ & 0.014 & $2.90 \pm 0.56$ \\
\hline BmiR41 & 0.000 & $-0.53 \pm 0.01$ & 0.586 & $0.18 \pm 0.30$ \\
\hline *miR42 & 0.293 & $-0.30 \pm 0.21$ & 0.013 & $-0.48 \pm 0.11$ \\
\hline *miR53 & 0.429 & $-0.24 \pm 0.25$ & 0.001 & $-0.52 \pm 0.06$ \\
\hline *miR56St & 0.531 & $-0.25 \pm 0.33$ & 0.005 & $-0.62 \pm 0.05$ \\
\hline BmiR66 & 0.040 & $0.70 \pm 0.20$ & 0.091 & $1.01 \pm 0.41$ \\
\hline "miR71 & 0.396 & $-0.29 \pm 0.28$ & 0.011 & $-0.62 \pm 0.06$ \\
\hline$\beta^{*} m i R 785$ & 0.036 & $-0.54 \pm 0.10$ & 0.002 & $-0.52 \pm 0.03$ \\
\hline BmiR786 & 0.002 & $-0.65 \pm 0.06$ & 0.437 & $-0.21 \pm 0.25$ \\
\hline "miR786St & 0.700 & $0.18 \pm 0.40$ & 0.022 & $-0.45 \pm 0.07$ \\
\hline BmiR793 & 0.031 & $1.21 \pm 0.22$ & 0.685 & $-0.07 \pm 0.15$ \\
\hline BmiR794 & 0.029 & $-0.54 \pm 0.14$ & 0.242 & $-0.26 \pm 0.16$ \\
\hline
\end{tabular}


Table 2 | The effect of parental post-embryonic nicotine exposure on miRNA expression levels in L4 worms belonging to the F2 generation. (* and $\beta$ denote $p$-values $<0.05$ in comparison to control)

\begin{tabular}{|c|c|c|c|c|}
\hline & \multicolumn{2}{|c|}{ F2 Low } & \multicolumn{2}{|c|}{ F2 High } \\
\hline & $P$ value & $\mathrm{MFC} \pm \mathrm{SE}$ & $P$ value & $\mathrm{MFC} \pm \mathrm{SE}$ \\
\hline$* \mathbf{m i R} \mathbf{1}$ & 0.636 & $0.24 \pm 0.44$ & 0.005 & $2.00 \pm 0.35$ \\
\hline *miR 1828 & 0.841 & $0.06 \pm 0.26$ & 0.046 & $-0.46 \pm 0.10$ \\
\hline *miR221 2St & 0.696 & $0.10 \pm 0.23$ & 0.001 & $0.50 \pm 0.02$ \\
\hline${ }^{*}$ miR2218a & 0.843 & $0.07 \pm 0.33$ & 0.013 & $0.96 \pm 0.11$ \\
\hline *miR2220 & 0.137 & $-0.40 \pm 0.17$ & 0.001 & $0.75 \pm 0.09$ \\
\hline$\beta *$ miR232 & 0.000 & $-0.61 \pm 0.05$ & 0.015 & $-0.59 \pm 0.07$ \\
\hline "miR235 & 0.003 & $0.64 \pm 0.10$ & 0.921 & $0.03 \pm 0.27$ \\
\hline$\beta^{*} \mathbf{m i R 2 3 9 a}$ & 0.006 & $-0.57 \pm 0.11$ & 0.000 & $-0.69 \pm 0.05$ \\
\hline$\beta * \mathbf{m i R 2 4 0}$ & 0.012 & $-0.61 \pm 0.07$ & 0.001 & $-0.59 \pm 0.02$ \\
\hline$\beta^{*}$ miR244 & 0.000 & $-1.00 \pm 0.00$ & 0.000 & $-1.00 \pm 0.00$ \\
\hline BmiR255 & 0.009 & $-0.50 \pm 0.05$ & 0.970 & $0.00 \pm 0.11$ \\
\hline BmiR5 1 & 0.043 & $0.55 \pm 0.19$ & 0.435 & $0.29 \pm 0.29$ \\
\hline$\beta *$ miR53 & 0.000 & $-1.00 \pm 0.00$ & 0.000 & $-1.00 \pm 0.00$ \\
\hline BmiR790 & 0.041 & $0.48 \pm 0.16$ & 0.054 & $0.35 \pm 0.08$ \\
\hline$\beta *$ miR80 & 0.027 & $0.81 \pm 0.14$ & 0.035 & $0.57 \pm 0.11$ \\
\hline "miR800 & 0.110 & $0.38 \pm 0.14$ & 0.049 & $0.52 \pm 0.19$ \\
\hline
\end{tabular}

0.5 folds $(\mathrm{p}=0.009)$ in grand-offspring of $20 \mu \mathrm{M}$-treated parents. On the other hand, the highest upregulation was observed for miR-1 $(2.0, \mathrm{p}=0.005)$ followed by miR-2218a $(0.9, \mathrm{p}=0.013)$, miR-2220 $(0.8, \mathrm{p}=0.001)$ and $\mathrm{miR}-800$ and $\mathrm{miR}-2212 *(0.5, \mathrm{p}=0.049$ and $\mathrm{p}=$ 0.001 , respectively) leaving miR- 1828 to be uniquely downregulated by 0.5 folds $(\mathrm{p}=0.046)$ in grand-offpring of parents treated with high nicotine dose (20 mM) (Figure 1b; Table 2).

Investigating the inter-generational effect of nicotine. Fourteen miRNAs were affected in more than one generation (Figure 1c). We checked for miRNAs whose expression was altered in more than one generation and found that indeed some miRNAs were commonly affected. Four miRNAs were altered in both F0 and F1 worm populations in response to direct and indirect nicotine treatment, respectively. As shown in Figure 2, miR-242 and miR-1829b were upregulated (1.0 fold) in F0 worms exposed to high nicotine concentration, while both were downregulated $(-0.5$ folds $)$ in their offspring. miR-794 was downregulated ( 0.5 folds) in F1 originating from the low nicotine treatment group without being affected in the parents, while it was upregulated $(0.6)$ in the parents (F0) exposed to high nicotine concentration. Though the F0 parents exposed to $20 \mu \mathrm{M}$ nicotine were not associated with an alteration in miR-785, it was downregulated $(-0.5)$ in their F1 offspring. On the other hand, it reversed in response to the $20 \mathrm{mM}$ concentration as it was upregulated in F0 (1.0 fold) to become downregulated in their offspring by 0.5 folds.

The parents and the grand-offspring also shared four altered miRNAs in response to nicotine. Both miR-2220 and miR-800 were altered in both F0 parents treated with the high nicotine dose and their grand-offspring. The extent seemed to decrease from 3.4 to 0.8 folds in the case of miR-2220, while it remained similar (0.5-0.6 folds) for miR-800. miR- 80 on the other hand was upregulated (1.0 and 0.9 folds) in both F0 parents exposed to low and high nicotine concentrations and remained like so ( 0.8 and 0.6 folds) in their grand-offspring, respectively.

The F1 offspring and F2 grand-offspring were grown in nicotinefree environments. Our results showed that out of the total miRNAs differentially expressed across all generations, five were common to $\mathrm{F} 1$ and F2. miR-53 and miR-1828 were downregulated $(-0.5)$ in the F1 offspring of parents exposed high nicotine treatment group and continued to be so in the F2 grand-offspring $(-1.0$ and -0.5 , respectively). Opposite patterns were observed for miR-2212* and miR-1 which started as being downregulated in F1 $20 \mathrm{mM}$ treatment group to become upregulated in F2. The expression of miR-1, miR-
$2218 \mathrm{~s}$ and miR-2212* was increased by almost 2.0 and 1.0 folds, respectively from $\mathrm{F} 1$ to $\mathrm{F} 2$ generations, both of which originated from parents and grandparents exposed to the high nicotine concentration. No altered miRNAs were shared between F1 and F2 worms from parents and grandparents exposed to the low nicotine concentration.

Interestingly, the expression of only one miRNA was computed to be statistically significant across all generations. It appears that miR255 was upregulated by more than 1.0 fold in F0 parents exposed to the high nicotine concentration. Neither this pattern, nor its inverse was observed in the succeeding generations. However, our data shows the miR-255 was downregulated ( -0.7 and -0.5 folds) in both F1 and F2 generations of parents and grandparents exposed to the lower nicotine concentration during its postembryonic development. Taken collectively, we report that fourteen miRNAs (6\%) were differentially altered in response to nicotine in at least two generations.

Seventy two miRNAs were differentially regulated across all generations. We investigated the patterns of miRNA expression levels across all generations in response to post-embryonic nicotine exposure in C. elegans. We compiled a list of all miRNAs whose expression was altered by at least 0.5 folds $(\mathrm{p}<0.05)$ in at least one generation. As a result, the list included seventy two miRNAs which represent $31 \%$ of all miRNAs tested. We constructed a heat map based on the Mean Fold Change (MFC) values for both $20 \mu \mathrm{M}$ (Low) and $20 \mathrm{mM}$ (High) nicotine treatment group across all generations (Figure 3). Different columns represent MFCs of six groups and are relative to control ( $0 \mu \mathrm{M}$ nicotine). Then, unsupervised hierarchical clustering based on Euclidean distance and complete linkage was performed and included six groups. For optimal visualization of the patterns, leaf ordering was done for both miRNAs and samples. As shown on the map, expression patterns for treatment groups belonging to the same generation were mostly similar to one another when compared to other generations. Thus, they were closely ordered next to one another. Also, we noticed another general pattern where worms exposed to the $20 \mathrm{mM}$ (high) nicotine treatment group as well as their F1 progeny had more altered miRNAs than those belonging to the lower nicotine concentration. Such was evident in the colored cells relative to those that were black (i.e. red: upregulated, green: downregulated, black: no change). However, the situation was different in the F2 generation where grand-offspring worms from parents exposed to high and low nicotine doses had similar expression patterns. Interestingly, our data showed that 


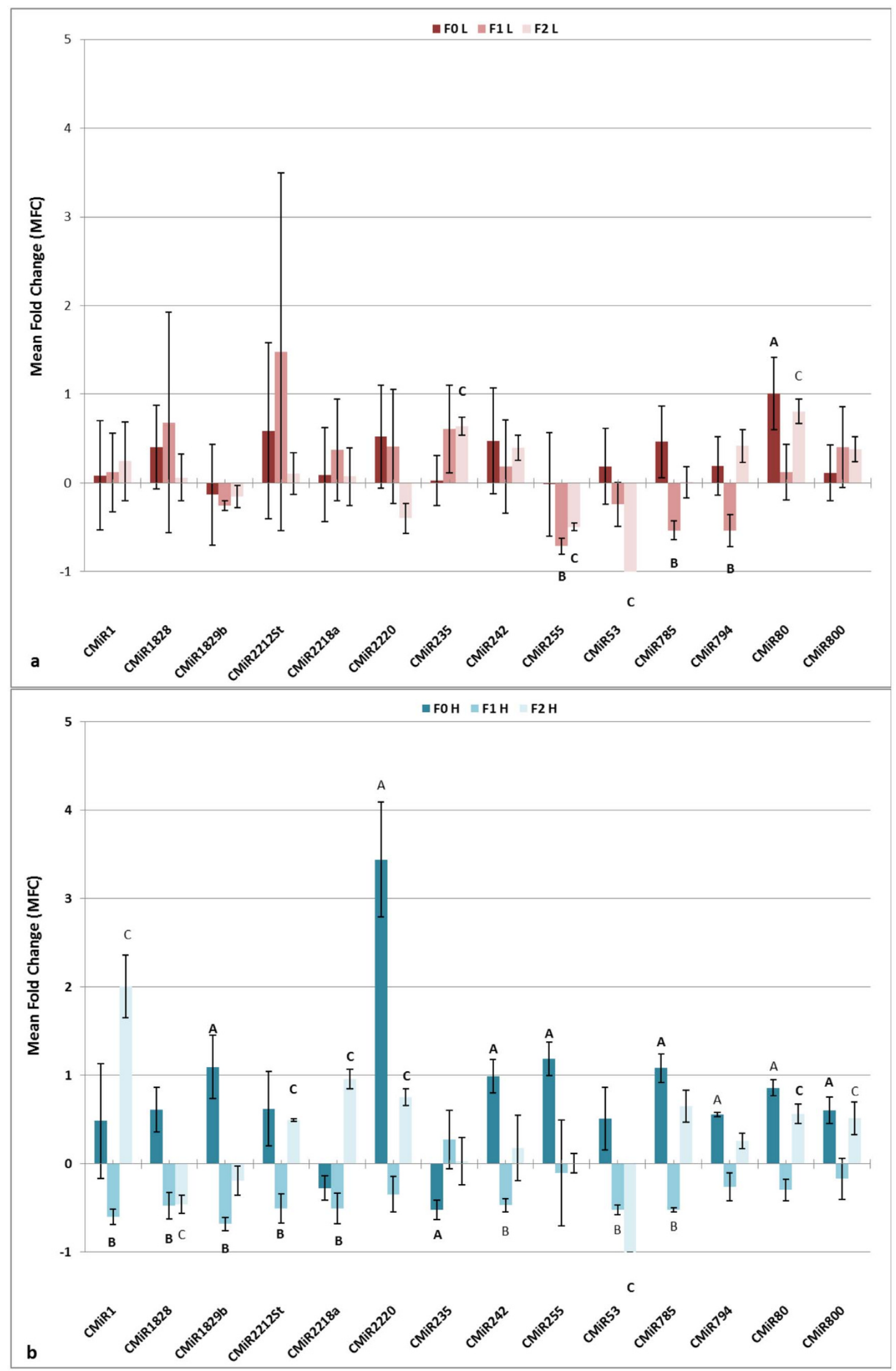

Figure $2 \mid$ Nicotine altered the expression levels of 14 miRNAs common to at least two generations. Different colors represent different treatment groups belonging to each of the three generations. From Left to right, bars represent Mean Fold Changes (MFC) for miRNAs belonging to (a) F0 L, F1 L, F2, L, and (b) F0 H, F1 H, and F2 H. L stands for low, and H stands for high nicotine dose. Data labels A, B, and C denote p-values < 0.05 in comparison to control in F0, F1, and F2, respectively. 


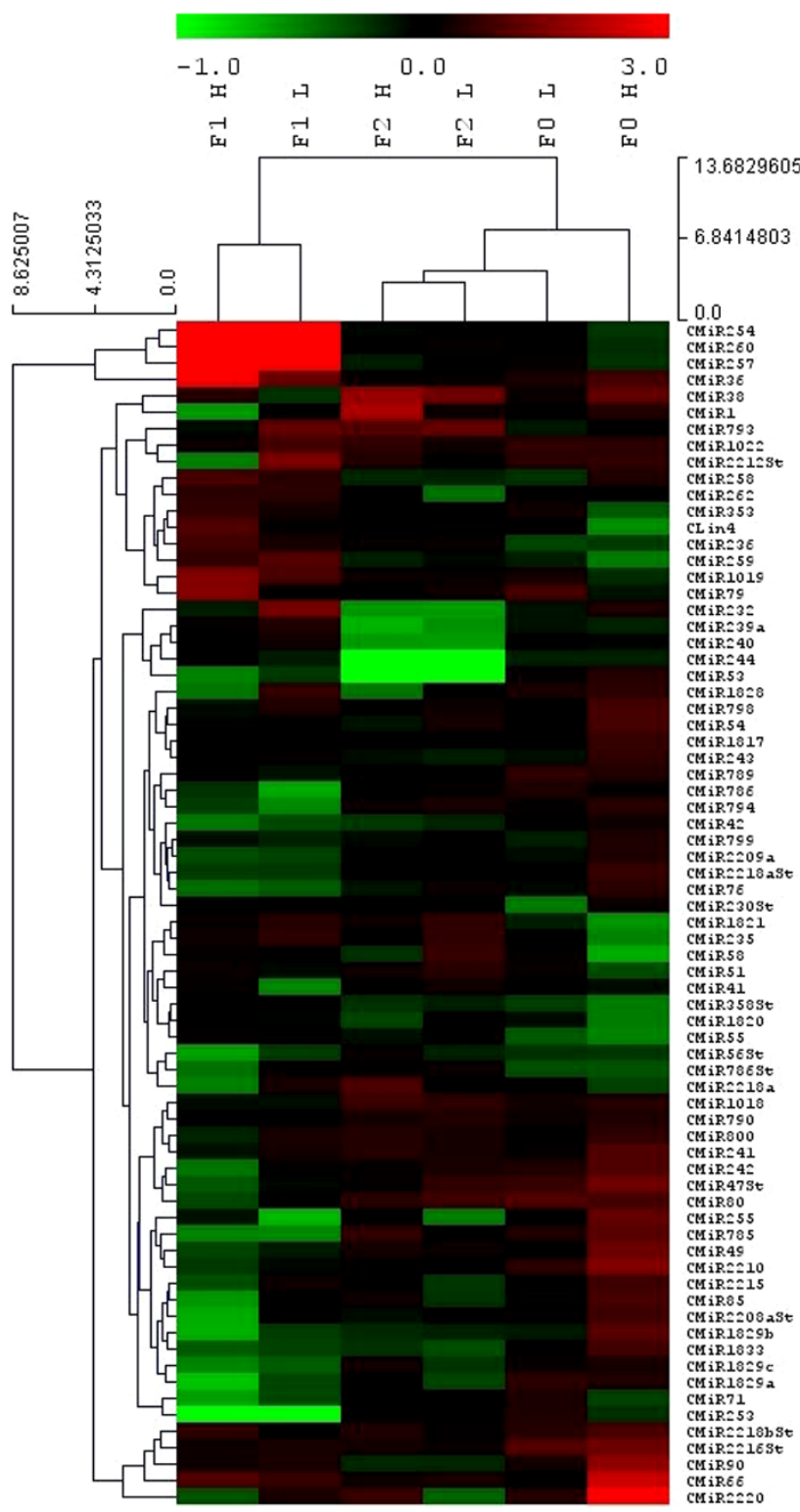

Figure $3 \mid$ Nicotine exposure limited to L4 of F0 generation caused differential clustering in three L4 generations in C. elegans (N2). Unsupervised hierarchical clustering based on Euclidean distance and complete linkage with optimization was performed for samples (3 biological replicates per treatment group) and miRNAs. Each cell represents a MFC compared to control (Mean Fold Change: $2(\Delta \Delta \mathrm{CT})-$ $1)$. In the figures, color red, green, and black represent up-regulation, down-regulation and no change with respect to control, respectively. Graph was done using Mev software ${ }^{40}$.

worms exposed to the lower nicotine concentration in F0 parents clustered with both F2 grand-offspring treatment groups. On the other hand, both groups in F1 had a different overall miRNA expression pattern and thus clustered separately from F0 and F2.

The fold changes of the seventy two miRNAs were also subjected to unsupervised optimized clustering. With 8.6 as a cutoff distance, results showed two clusters, one that included four miRNAs (miR254, miR-260, miR-257, and miR-36), while the other included sixty eight. Decreasing the cut-off distance by half to 4.3 resulted in one additional cluster of five miRNAs (miR-2220, miR-66, miR-90, miR2216*, and miR-2218b*).

For a more qualitative assessment of the expression changes across the three generations, we performed further analysis on the seventy two miRNAs that showed differential alteration in at least one generation. The exact values for the expression levels have been thoroughly discussed above. Hence, we provided further elaboration to study the effect of nicotine in a dose and generation-dependent manner. As shown in the radar graphs (Figure 4), each concentric ring represents respective fold changes with zero as no change. miRNAs were ordered alphabetically to allow for comparison between low and high treatment groups across generations. In F0, the direct nicotine effect was more dramatic in the high versus the low concentration treatment. As an overall, the pattern seen in response to the $20 \mu \mathrm{M}$ nicotine concentration was circular and generally uniform, bordering the zero-fold-rim, and did not exceed 1.0 fold change in either direction. On the other hand, the circular pattern was no longer uniform in worms treated with the $20 \mathrm{mM}$ nicotine dose. Much more miRNAs were increased by $>1.0$ folds, and the pattern borders mostly intersected with 1.0 fold-rim. In F1, though nicotine was no longer in direct contact, the progeny of worms exposed to the low nicotine concentration had more altered miRNAs than their parents. The pattern is no longer circular and was reformed by dramatic up and downregulations that reached the $4^{\text {th }}$ rim in the positive direction, and the $1^{\text {st }}$ rim in the negative direction, respectively. A more defined shape was observed in the offspring of parents exposed to the high nicotine concentration as it had a star-shaped pattern in the F1 generation. The increase in the downregulated miRNAs was similar to offspring of the low nicotine concentration. However, the main shifts in expression levels were the doubled upregulations in three out of four miRNAs (miR-260, miR254, miR-36, and miR-257). Finally in F2, the pattern went back to become circular. Nonetheless, unlike the F0 generation, the circular pattern was similar in grand-offspring originating from grandparents treated with either nicotine doses. The circular pattern generally overlapped with the F0 $20 \mu \mathrm{M}$ treatment group, and this was consistent with results shown in the heat map after hierarchical clustering.

Investigating the nicotine potential effectors downstream of the altered miRNAs. Most miRNAs have pleiotropic functions. It is known that one gene can be targeted by up to hundreds of miRNAs. Vice versa, one miRNA can target hundreds of genes. Therefore, to understand processes that are mostly affected by nicotine treatment, we limited our analyses on the fourteen miRNAs that showed differential expression in at least two generations (Figure 2). Thus, a list of genes predicted to be targeted by miR-242, miR-1829b, miR-785, miR-1, miR-2218a, miR-53, miR-235, miR-80, miR-2220, miR-800, miR-255, miR1828, miR-2212*, and miR-794 was compiled. The list included 2462 unique gene suspects. Two genes ( $r b c-1$ and egl-10) were predicted to be targeted by five miRNAs. Based on annotations from wormbase ${ }^{47}, r b c-1$ might be involved in proton-pump translocation, while egl-10 has a role in egg laying and regulation of certain signaling pathways. Four miRNAs were predicted to regulate each of F10D2.10, miz-1 (zinc-finger transcription factor), cyp-23A1 (xenobiotic metabolism), C50H11.8, and tag-97.44 genes were predicted to be targeted by three miRNAs. 348 were expected to be common to two miRNAs included in our analyses. The remaining genes were not considered as common targets to the fourteen miRNAs included in the target prediction analysis.

To investigate whether each miRNA is involved in particular pathways, a gene list for each of the fourteen miRNAs was used as an input for DAVID analysis. Based on the percentage of genes belonging to a certain process, an enrichment value is computed coupled with p-values (Fold enrichment, p-value). Enrichments manifested in KEGG pathways were associated with only four miRNAs. miR1828 might have a role in the Wnt-signaling pathway $(3.3, \mathrm{p}=$ 0.058). miR-80 was predicted to target genes involved in Notch $(6.0, p=0.025)$, MAPK $(3.0, p=0.049), \operatorname{ErbB}(4.0, p=0.082)$ 

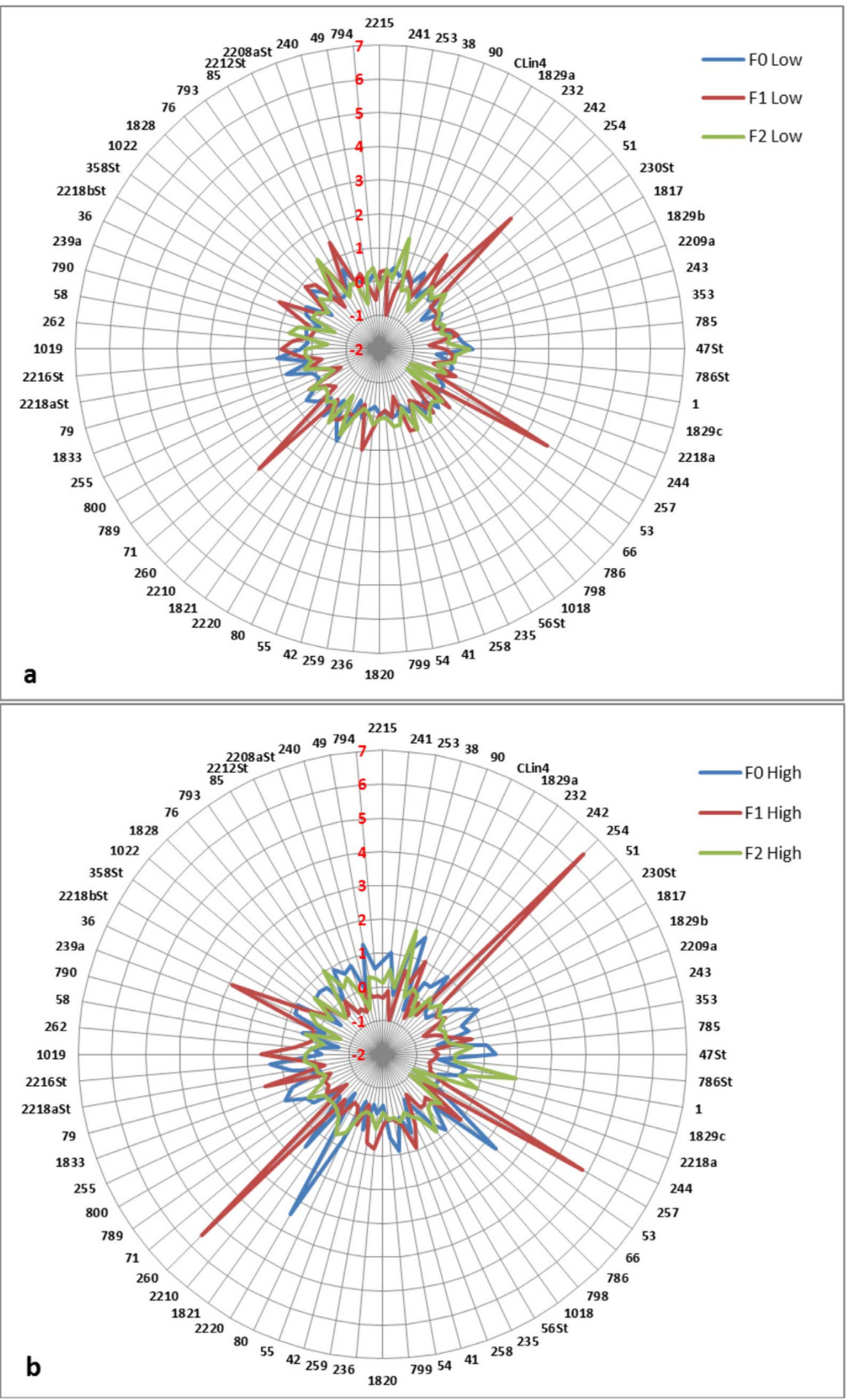

Figure $4 \mid$ Radar graph showing general patterns of miRNA MFCs across generations in response to nicotine treatment limited to the post-embryonic stage of the parent F0 generations in C. elegans (N2). The MFCs of 72 miRNAs were used for the low treatment groups (20 $\mu \mathrm{M})$ (a) and high treatment groups $(20 \mathrm{mM})(\mathrm{b})$. Each concentric rim represents 1 fold increase or decrease $(2(\Delta \Delta \mathrm{CT})-1)$ from 0 . Different generations are presented by different colors. The input miRNA list had the same order among each treatment group (low and high nicotine concentration) to allow for comparisons. 
signaling pathways. Also, $\operatorname{MAPK}(2.4, \mathrm{p}=0.095)$ as well as endocytosis $(3.1, \mathrm{p}=0.006)$, and pyrimidine metabolism $(2.9, \mathrm{p}=0.016)$ might be regulated by miR-785. Finally, miR-1 was estimated to target signaling pathways such as the phosphatidylinositol system (5.7, $\mathrm{p}=0.003)$, lysosome $(2.8, \mathrm{p}=0.02)$, oxidative phosphorylation (2.2, $\mathrm{p}=0.027)$, Wnt $(2.7, \mathrm{p}=0.035)$, inositol phosphate metabolism (5.2, $\mathrm{p}=0.036)$, and mTOR signaling pathways (3.9, $\mathrm{p}=0.075)$.

Since the miRNA annotation research is still in the juvenile stages, we were interested in investigating possible relationships between all the genes predicted to be targeted by the fourteen miRNAs. In order to do that, we used GOrilla software which provides a DAG (Directed Acyclic graph) that shows relationships among enriched pathways predicted to be regulated. The input for GOrilla was ranked. To prepare the ranked list, we performed functional annotation clustering for the 2462 genes through DAVID with highest stringency classification. The output from DAVID included 120 clusters arranged in decreasing order of enrichment values. Genes belonging to clusters with enrichment values greater than two were considered and were ranked in decreasing order (from most to least enriched). The latter list was composed of 141 ranked genes and was used for input in GOrilla. The highest enrichments belonged to positive regulation of translation $\left(10.9, \mathrm{p}=10^{-3}\right)$ followed by macromolecule modifications $\left(6.7-10, \mathrm{p}<10^{-3}\right)$ (e.g. peptidyl-lysine and amino acid modification, protein metabolism). The fourteen miRNAs were computed to targeted metabolic and biosynthetic regulatory genes $(4.6, \mathrm{p}<$ $\left.10^{-9}\right)$. Transcriptional control as well as RNA, nitrogen and nucleobase metabolism were also highly enriched $\left(4.6, \mathrm{p}<10^{-9}\right)$. Processes like taxis and chemotaxis, axon guidance had an enrichment value of 2.9 with $\mathrm{p}<10^{-7}$. Targeted processes like response to external ( $\mathrm{p}<$ $\left.10^{-5}\right)$ and chemical stimuli $\left(\mathrm{p}<10^{-3}\right)$, cellular development $(\mathrm{p}<$ $\left.10^{-3}\right)$ as well as phosphorylation $\left(\mathrm{p}<10^{-3}\right)$ had enrichment values ranging from 2.1 to 2.7 (Figures 5 and $6 \mathrm{a}$ ).

Then, a bottom-top approach was followed starting with matching genes of every enriched pathway to their predicted regulatory miRNAs. Based on GOrilla predictions (Figures 5 and $6 \mathrm{ab}$ ), miR242 was not involved in any of the enriched processes. miR-53 and miR-1829b targeted genes involved in molting cycle and locomotion. In addition to these two processes, miR- 800 might regulate cellular development, and response to stimulus. Interestingly, targets of miR785 were restricted to nitrogen, nucleobase, and RNA metabolic processes, biosynthesis, and transcriptional regulation. On the other hand, miR-235 covered many of the enriched processes, except for taxis, and other functions related to protein metabolism and modifications. Six of the remaining miRNAs (i.e. miR-2218s, miR-794, miR-255, miR-2212*, and miR-80) might be working redundantly as they covered all processes except for those related to protein metabolism and some modifications. Finally, the last two miRNAs were the most pleiotropic and were anticipated to fine-tune genes encoding of the enriched functions except for cellular development and peptidyl lysine modification as was the case for miR-2220 and miR-1, respectively.

Pathways with the highest enrichment values and statistical significance. We narrowed our analysis further by focusing on select pathways predicted by GOrilla to be altered in response to nicotine (Figure 7a). Enrichment values $>3.0$ and p-values $<10^{-9}$ were used as cutoff criteria. Out of the fifty two overall pathways, only fourteen were then chosen for downstream analysis. 25 genes involved in those pathways were then extracted and used as input for gene functional annotation through DAVID. Such was run with highest stringency and resulted in two clusters. The first one, including unc-30, skn-1, nhr-49, fos-1, nfi-1, and vab-3, had an enrichment value of 21.4. The second one, including daf-4, daf-1, $c m k-1$, pek-1, and sma-6, had a lower enrichment value of 11.3. As detailed in Table 3, their functions include response to stress, development and differentiation, behavior such as egg laying and locomotion. These results were consistent with the overall target prediction discussed in the previous paragraphs.

Analysis through GOrilla provided a general idea about the enriched pathways across the three generations; however, it might have masked differences between individual generations. We wanted to investigate common effectors between only two generations. For F0-F1 pair, target prediction was done for five differentially altered miRNAs (miR-242, miR-785, miR-1829b, miR-794, and miR-255). For F0-F2 pair, also five miRNAs were used (miR-255, miR-2220, miR-80, miR-800, and miR-235). For F1-F2 pair, (miR-1, miR2218a, miR-2212*, miR-1828, miR-53, and miR-255) were used for target prediction. The resulting gene lists included 1047, 1046, and 1149 genes for F0-F1, F0-F2, and F1-F2 pairs, respectively. The latter were individually run through DAVID for functional annotation clustering with highest stringency. Only clusters with enrichment values $>3.0$ were considered and the corresponding genes were extracted. Based on these criteria, F0-F1 pair didn't show any

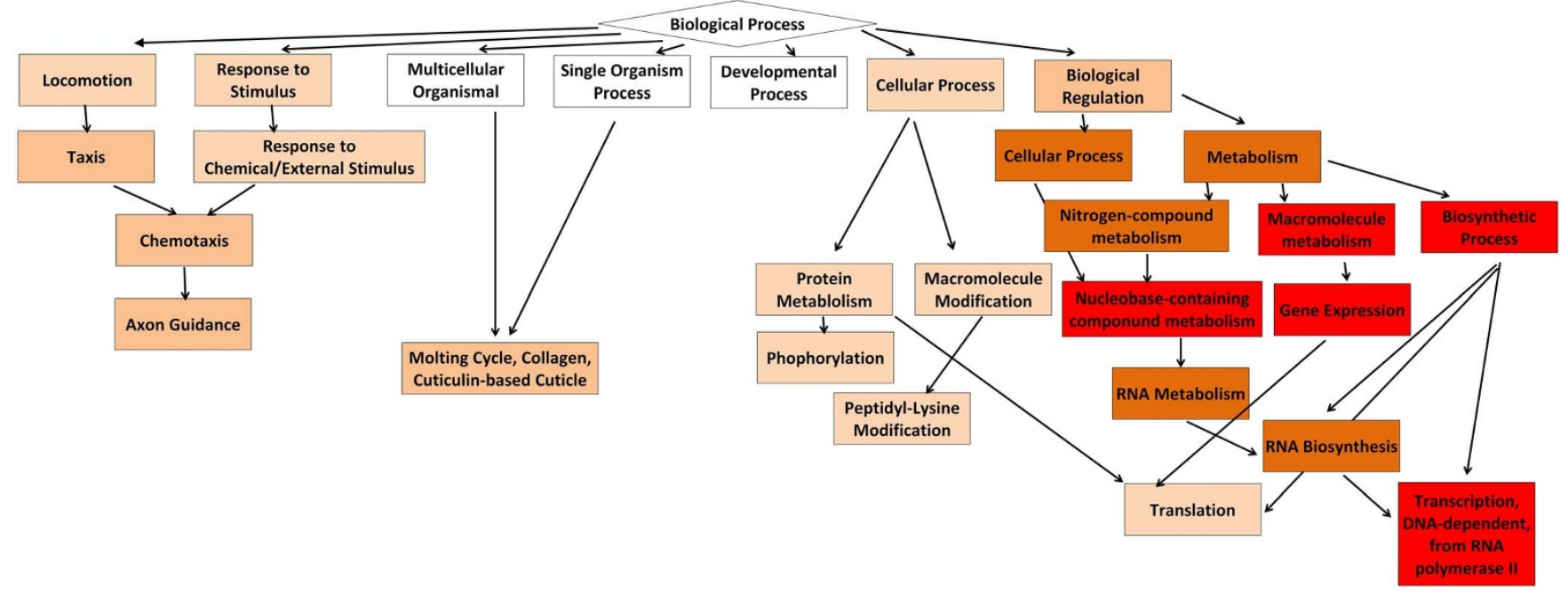

Figure $5 \mid$ Directed acyclic graph (DAG) performed by GOrilla. 14 miRNAs that were differentially expressed in at least 2 generations were used for target prediction. Only genes belonging to enriched clusters in DAVID were used to prepare the single gene ranked list for GOrilla input as described in the text. The Graph shows the relationships among the enriched pathways targeted by miRNAs altered in response to nicotine treatment. Colors represent P-values. From white to orange/red, p-values range from $>10^{-3}$ to $<10^{-9}$. 


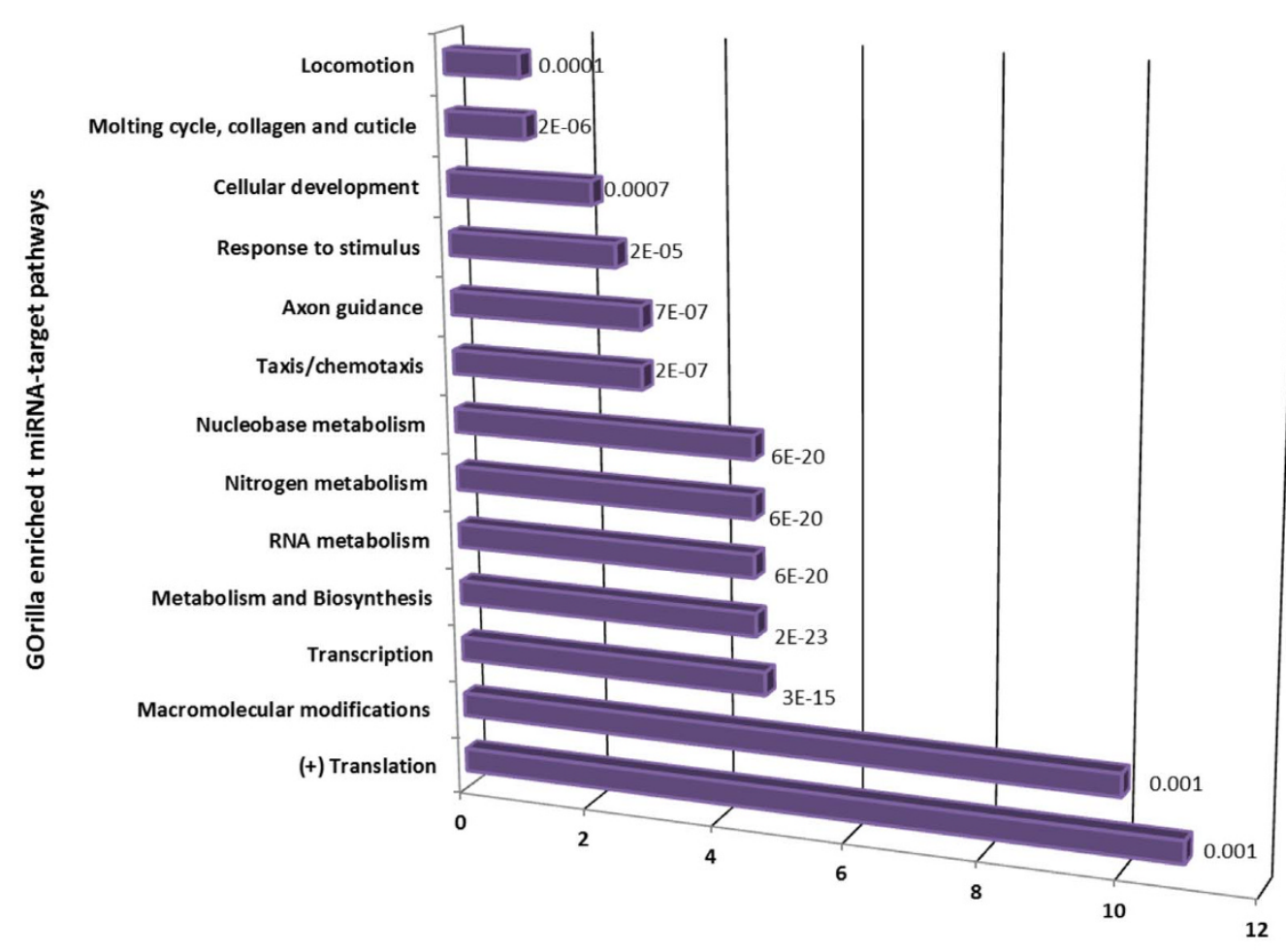

Enrichment Value

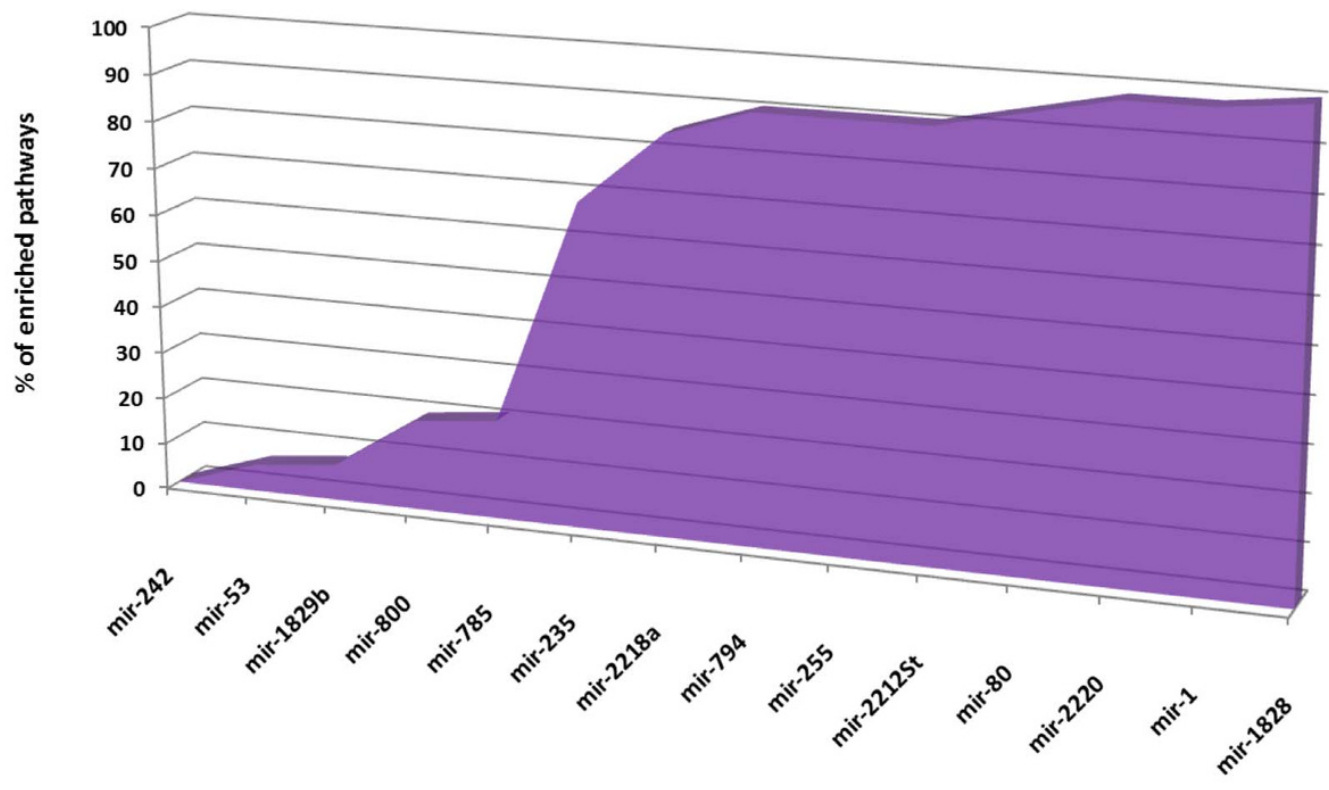

b

Figure 6 Analyzing the roles of the 14 commonly altered miRNAs in the enriched pathways. (a) A summary of the pathways predicted to be altered in response to nicotine treatment across the three generations. The data labels represent the respective p-values for each enriched process. (b) Variation in miRNA regulation. Some pathways are commonly regulated by 2 or more miRNAs while others are specific to 1 miRNA. On the other hand, there was an apparent difference in the \% of pathways predicted to be regulated by individual miRNAs. 


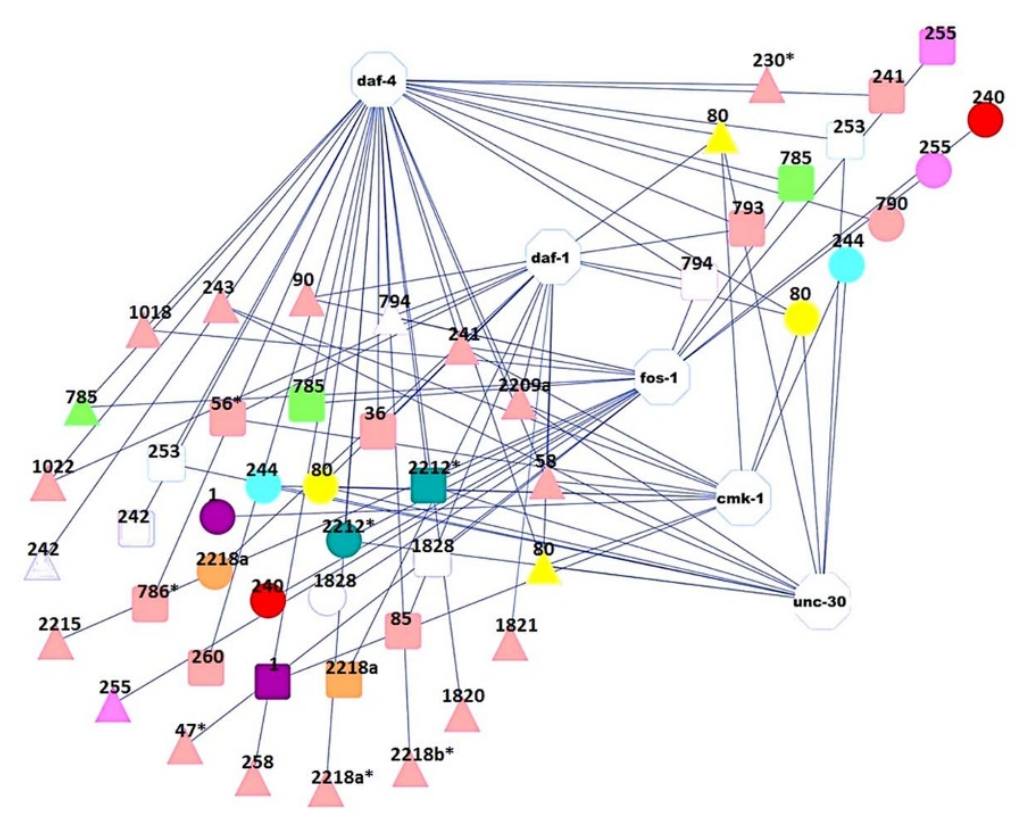

a

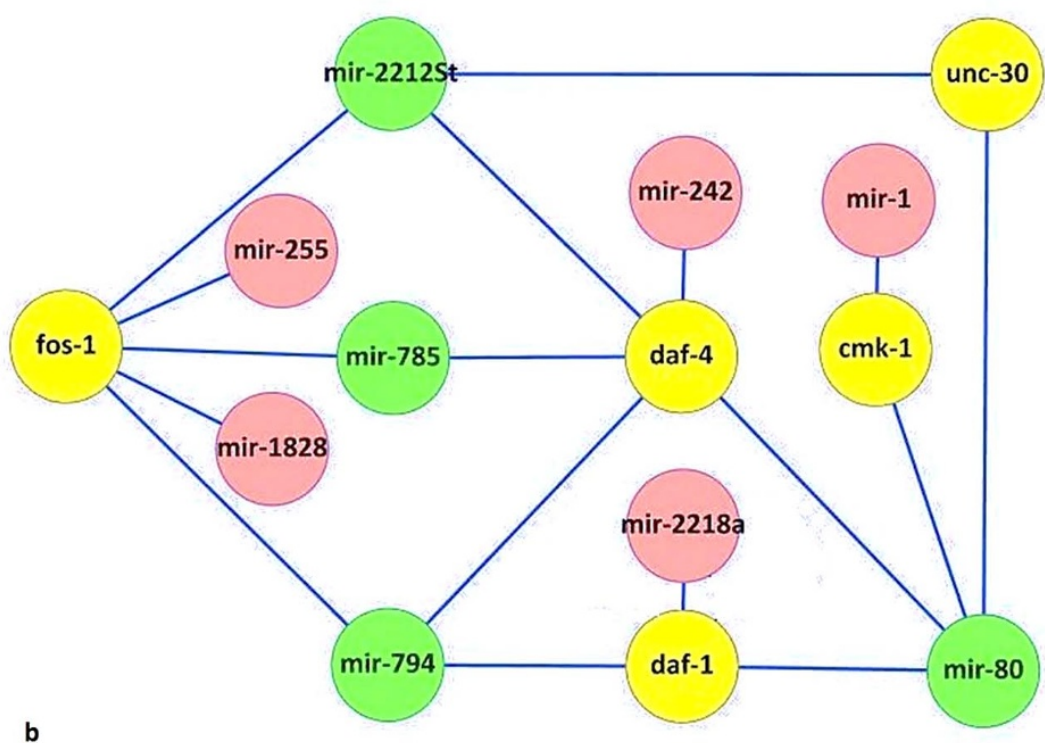

b

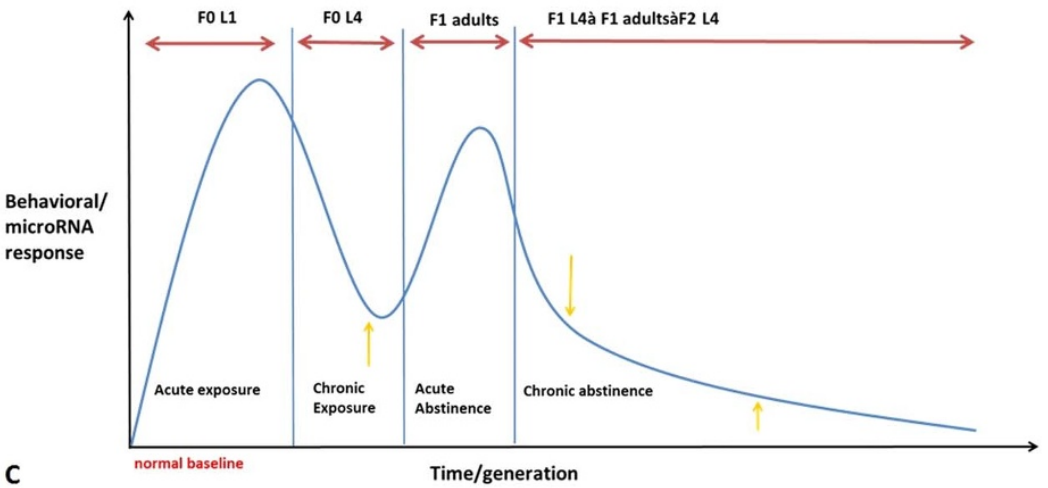

Figure $7 \mid$ Nicotine exerts its transgenerational effect via five enriched gene candidates. (a) A network showing relationships between differentially altered miRNAs with gene candidates in each generation. Triangles denote worms of the F0 generations, while squares and circles denote worms of the F1 and F2 generations, respectively. The five genes candidates are in hexagons in the middle. The low concentration treatment groups are shown on the up-right side, while the high concentration treatment groups are shown in the left bottom side. (b) A simplified network showing interactions between only commonly altered miRNAs and the candidate genes. Candidate genes are in yellow. miRNAs targeting more than one gene are in green. (c) A hypothesized model to explain the nicotine-induced miRNA expression profiles and behaviors as a function of duration of exposure or abstinence and previous parental life experiences. 
Table $3 \mid$ A summary of genes clustered with high enrichment using Gene functional annotation via DAVID. Worm annotations were obtained from WormBase ${ }^{47}$

Gene Group 1

Enrichment Score: 21.4

Official gene symbol

Function

unc-30

(UNCoordinated)

skn-1

(SKiNhead)

- homeodomain-containing protein

- orthologous to the Pitx family of homeodomain transcription factors

- controls the terminal differentiation of all 19 type D GABA-ergic motor neurons during development

- bZip transcription factor orthologous to the mammalian Nrf (Nuclear factor-erythroid-related factor) transcription factors

- required for specification of the EMS blastomere, a mesendodermal precursor that gives rise to pharyngeal, muscle, and intestinal cells during early embryogenesis

- functions in the p38 MAPK pathway to regulate the oxidative stress response and in parallel to DAF-16/FOXO in the DAF-2-mediated insulin/IGF-1-like signaling pathway to regulate adult lifespan

nhr-49

- nuclear hormone receptor (NHR) related to the mammalian HNF4 (hepatocyte nuclear factor 4) family of NHRs

(Nuclear Hormone Receptor

- key regulator of fat metabolism and lifespan by regulating induction of beta-oxidation genes upon food deprivation

family)

and activation of stearoyl-CoA desaturase in fed animals, respectively

- activates transcription in conjunction with the MDT-15 mediator subunit with which it physically interacts

fos- 1

(FOS B-Zip transcription

factor homolog)

- encodes two basic region-leucine zipper (bZip) transcription factors, FOS-1 A and FOS-1B, that are the sole $C$. elegans ortholog of the fos bZip transcription factor family

- required cell autonomously in the gonadal anchor cell for basement-membrane removal and subsequent anchor cell invasion of the vulval epithelium

nfi-1

(Nuclear Factor I family)

vab-3

(Variable ABnormal

morphology)

- encodes the C. elegans ortholog of the Nuclear Factor I (NFI) family of transcription factors in C. elegans

- required for locomotion, egg laying, pharyngeal pumping, and wild-type adult lifespan

- homeodomain protein (Pax-6 ortholog)

- required for proper patterning of anterior (head) hypodermal cells

- required for epidermal morphogenesis, epidermal cell fates, gonad cell migration and the development of sensory structures in the male tail

Gene Group 2

Enrichment Score: 11.3

Official gene symbol

Function

daf-4

- transmembrane serine/threonine kinase (sole ortholog of the type II transforming growth factor-beta (TGF-b)

(abnormal DAuer Formation) receptors)

- required for several biological processes, including entry into and exit from the dauer larval stage, body size determination, male tail patterning, egg laying, chemosensory neuron specification, and increased

thermotolerance

- regulates reproductive aging, via the TGF-beta Sma/Mab pathway

daf-1

- TGF-beta type I receptor homolog

(abnormal DAuer Formation) - required for the regulation of daver formation by environmental signals through the ASI chemosensory neuron

cmk- 1

(CaM Kinase)

pek-1

(human PERK kinase

homolog)

sma-6

(SMAII)
- Ca +2 /calmodulin-dependent protein kinase I (CaMK1)

- required, cell autonomously and downstream of the cyclic nucleotide-gated channel TAX-4, for several aspects of AFD thermosensory neuron differentiation, including expression of the gcy-8 guanylyl cyclase and nhr-38 nuclear hormone receptor genes and morphology of the AFD sensory endings

- required for normal thermosensory behavior

- positively regulate the transcriptional activity of endogenous CREB

- predicted transmembrane protein kinase (orthologous to human eukaryotic translation initiation factor 2-alpha kinase 3 (EIF2AK3))

- strongly expressed in intestinal cells

- required for the unfolded protein response (UPR) that counteracts cellular stress induced by accumulation of unfolded proteins in the endoplasmic reticulum (ER)

- may phosphorylate elF2alpha and inhibit protein synthesis in response to endogenous ER stress

- serine/threonine protein kinase (ortholog of type I TGF-beta receptors)

- required for regulating body length and for proper development of the male tail

- regulates reproductive aging

- sufficient for body length regulation clusters. F0-F2 pair had one enriched cluster (regulation of biosynthetic process, $\mathrm{E}=5.9$ ) and included 16 genes. The $\mathrm{F} 1-\mathrm{F} 2$ pair had three enriched pathways involved in the regulation of biosynthesis and transcription $\left(\mathrm{E}_{\mathrm{avg}}=4.2\right)$ and included a total of 15 genes. The absence of enriched pathways from target genes predicted to be regulated by miRNAs common to the parents and their offspring in the F0 and F1 generations suggests that different processes were altered in F0 versus F1 generations. This result is complimentary to 
the hierarchical clustering where the distance between F0 and F1 was the highest, suggesting least commonalities in miRNA expression patterns.

unc-30, fos-1, daf-4, daf-1, cmk-1, sma-1, and nfi-1 were common genes among the outputs from the above two approaches. Of the latter, only unc-30, fos-1, daf-1, daf-4, and $c m k-1$ were targeted by differentially altered miRNAs in at least five of the six treatment groups across all generations. daf- 4 was predicted to be targeted by twenty two miRNAs, daf- 1 and fos- 1 were predicted to be targeted by fourteen and twelve miRNAs, respectively, while $c m k-1$ and unc-30 were targeted by eight and seven miRNAs, respectively (Figure 7a). Figure $7 \mathrm{~b}$ depicts these five genes targeted by one or more of the fourteen commonly altered miRNAs (Figure 7, Table 3).

\section{Discussion}

Both genetics and environment determine the phenotype. Stress exposed during early development till adolescence has enduring effects (e.g. neuro-remodeling, sensitivity to drugs of abuse). A study showed that chronic nicotine exposure during adolescence but not adulthood lead to long-lasting alterations in the cognitive performance in rats ${ }^{15}$. Epigenetic modifications might be mediators of these short or long-term, and even transgenerational changes ${ }^{48}$. To be considered as truly transgenerational, effects should remain evident in at least three or four generations depending on the ancestral exposure period. Those that occur in impregnated individuals should leave a mark on four or more generations, while experiences limited to ancestral postnatal period or non-gravid adults impact three or more generations ${ }^{49}$. In our study, post-embryonic nicotine exposure was restricted to F0 ancestors prior to their sexual maturity. This exposure was associated with an altered miRNA expression profile that remained evident in three generations. Therefore, it modeled a true transgenerational effect.

Our results support the "two-hit" model proposed by Crews et $\mathrm{al}^{48}$. The first "hit" was the chronic nicotine exposure during the post-embryonic stage in C. elegans. The second "hit" was the withdrawal-associated stress experienced during gestation. Together, they caused alterations in the subsequent progeny (larvae) at the miRNA level, the focus of our study. Interestingly, some of the effects were shared while others were context dependent (e.g. nicotine dose, exposure vs. abstinence, acute vs. chronic, developmental period) (Figure 7c).

Concentration specific patterns. Chronic nicotine treatment was limited to about 30 hours from L1 to L4 in F0 generation worms. The subsequent generations were not in direct contact with nicotine. Despite that, nicotine affected miRNA profiles in a dose-dependent manner. In worms treated with the low nicotine concentration, the percentage of differentially expressed miRNAs remained similar across the generations (1-4\% of miRNAs). However, a decreasing pattern was observed in worms treated with the high nicotine concentration as the generations progressed. $16.5 \%$ of the miRNAs had disparate expression in response to the direct exposure to the high nicotine concentration. The percentage decreased to about $11 \%$ in their F1 offspring. In the grand-offspring (F2), only $5.2 \%$ of the miRNAs showed changes in their expression patterns. This implies that nicotine treated chronically and strictly during the adolescent stages of the parent generation had a long lasting effect that was still detected in the third generation. Moreover, the dose-dependent effect was also passed along the generations as the severity of withdrawal was proportional to the nicotine dose and duration of exposure $^{50}$. Unlike the high nicotine concentration, the low nicotine dose was pharmacologically active but asymptomatic. Such a dosedependent effect decreased but remained evident in each generation. The F0-initiated nicotine ripple faded as the generations progressed to reach a baseline adaptive response observed in worms succeeding from the $20 \mu \mathrm{M}$ (low) nicotine treatment group.
Generation versus nicotine concentrations. Some miRNA expression alterations were specific to one nicotine dose, but not the other, while the expression of other miRNAs was altered in response to both nicotine concentrations. The difference was mostly evident in the F0 generation where worms were in direct contact with nicotine. Only $2.5 \%$ were commonly affected in response to the low and high nicotine concentrations. However, the miRNA response almost tripled in F1 offspring as 9.7\% miRNAs were commonly responsive to both low and high nicotine concentrations. The percentage continued to increase in F2 grandoffspring as $37.5 \%$ of the miRNAs were commonly affected in response to both high and low grandparental nicotine exposure.

Generation specific patterns. $31.2 \%$ of the miRNAs were totally altered in response to both nicotine concentrations. As the generations progressed, the number of totally altered miRNAs decreased from $17.3 \%$ in $\mathrm{F} 0$ to $13.4 \%$ in $\mathrm{F} 1$ and finally became $6.9 \%$ in F2. Each generation had a pattern generally consistent between the two nicotine concentrations. About $72 \%$ of the miRNAs were upregulated in F0. However, most of the miRNAs (67\%) were downregulated in F1, while almost half of them (55\%) were upregulated in the F2 worm population.

How can we explain the opposite miRNA patterns observed in F1 while both F0 and F2 were similar? Nicotine exposure was limited to the F0 generation. Similar miRNA profiles were observed in F0 and F2, while F1 had an opposite pattern as shown in the heat map, and radar graphs. Revisiting our experimental settings, L4 larvae belonging to the F0 generation were chronically exposed to nicotine and thus were desensitized to it. The L4 larvae of the F1 generation were the progeny of F0 parents experiencing acute nicotine withdrawal. Finally, L4 larvae grand-offspring (F2) progressed from parents (F1) experiencing protracted withdrawal. This is depicted in Figure 7C where all three time points are elucidated by arrows.

It is crucial to recall two general nicotine-induced responses. The first is transient and results from either "acute" exposure or abstinence from nicotine. The second is adaptive and occurs after "chronic" treatment or abstinence from nicotine. Such might involve epigenetic modifications that stabilize gene expression in a dose and time-dependent manner in response to environmental changes. Thus, after adapting to a stimulus, an environmental shift back to "normality" is conceived as a new fluctuation. The system then responds transiently if the stimulus is temporary. Conversely, the response takes an adaptive, relatively permanent form in case the trigger is persistent. Thus, parent (F0) larvae model an adaptive state in response to chronic treatment. The perinatal period is vulnerable and sensitive to stress. It has been suggested that stress responses are transmitted through the germ line and are apparent in subsequent generations even in the absence of stressor ${ }^{48}$. To further support the latter, C. elegans maintains an epigenetic transcriptional memory through parental primordial germ cells ${ }^{51}$. Thus, grand-offspring (F2) larvae also model adaptation as they are the progeny of F1 parents experiencing chronic nicotine withdrawal. On the other hand, not only can chronic treatment followed by acute withdrawal disrupt a system, but contrasting symptoms can also arise after transitioning from acute to protracted withdrawal. With this in mind, the intermediate F1 generation does not model adaptation as their parents were experiencing acute withdrawal. Instead, they are sensitized and represent transience. This idea is clearly supported by an experiment done on rats where both direct nicotine exposure and chronic withdrawal were associated with a decrease in inhibitory control (i.e. addiction-related index). On the contrary, those rats undergoing acute withdrawal experienced improved inhibitory control ${ }^{52}$. Collectively, the aforementioned scenarios can partly explain the similarities between F0 and F2 in contrast to F1 (Figure 7c). 
Functional annotation of miRNAs whose expression was altered in response to nicotine. The miRNAs whose expression showed differential alterations in response to post-embryonic nicotine treatment in F0 belonged to many families. However, miR-51 and miR-80 families were more likely to mediate nicotine-induced effects. miR-51 family members are associated with a wide range of processes. Some of its members (i.e. miR-51, miR-52, and miR-5456) might regulate the developmental timing by antagonizing let-7 and lin-4. This family was also hypothesized to regulate miRISC activity which increased after the loss of miR-51 members and others like miR-239a and miR-244. The latter two were among the miRNAs that showed differential expression across generations. miR-51 family members are broadly and highly expressed ${ }^{53}$. Therefore further upregulation might hinder other miRNA activities by surpassing their miRISC complex binding ${ }^{54}$. Another abundant and constitutively expressed family is miR-58/80 family ${ }^{53,55,56}$. The deletion of all of miR-58 family members resulted in a sluggish worms with smaller body sizes. The miR-58 family members have a role in locomotion, body size and egg laying. Most of these traits are common with nicotine related phenotypes in C. elegans (e.g. paralysis in response to high nicotine concentration $)^{21}$. This might suggest a role of miR-58/80 family in nicotine molecular mechanism in the worm.

Eight major functional hubs were mapped through GOrilla. These included locomotion, response to stimulus, multicellular organismal process, single-organism process, developmental, cellular and metabolic processes, and biological regulation. The highest statistically significant enrichment values belonged to the biological regulation hub. The latter included regulation of transcription and gene expression, metabolism (e.g. RNA, nucleobase-containing compound), and biosynthesis pathways with enrichment values $>3$ and $\mathrm{p}$-values $<10^{-9}$. Similarities in pathway enrichment analysis were observed between F0 and F2, or F1 and F2 generations, but not $\mathrm{F} 0$ and $\mathrm{F} 1$. This is sensible when considering parents being under direct nicotine exposure, while their offspring originated from parents undergoing acute abstinence.

Of course, response to environmental stimuli systematically alters a myriad of factors. However, we funneled down our analysis and focused on five genes predicted to have a role in nicotine's mechanism of action across generations. The targets were regulated by the fourteen miRNAs whose expression levels were changed in more than one generation. We propose a model where daf-4, daf-1, fos$1, \mathrm{cmk}-1$, and $u n c-30$ are regulated by most of the fourteen miRNAs in a context dependent manner across generations. Based on annotations from WormBase ${ }^{47}$, daf- 1 could mediate the reception of the nicotine presence/absence as it is expressed in the chemosensory neurons. fos- 1 and $c m k-1$ mediate neuro-adaptation and remodeling in response to acute versus chronic exposure/withdrawal ${ }^{57-60}$. daf-4 might explain the downstream phenotypic changes as it is involved in exit from dauer larval stage, body size determination, male tail patterning, egg laying, chemosensory neuron specification, thermotolerance and reproductive ability. It is important to consider miRNAs as working as a system where synergistic, additive, as well as antagonistic effects arise and are affected by nicotine dose, duration of exposure, generation, as well as previous ancestral experiences. The net effect is a generation-specific phenotype, characterized by shared as well as unique features.

1. WHO. Tobacco, (2012) (Date accessed: 24/03/2012) http://www/who.int/ mediacentre/factsheets/fs339/en/.

2. WHO. World Health Statistics. Global Health Observatory, (2012) (Date accessed: 24/03/2012) http://www.who.int/gho/publications/world_health_ statistics/EN_WHS2012_Full.pdf.

3. USDHHS. The Health Consequences of Smoking: Nicotine Addiction. 1-618 (Center for Health Promotion and Education. Office on Smoking and Health, 1988).
4. CDC. Morbidity and Mortality Weekly Report, (2008) (Date accessed: 12/08/2012) http://www.cdc.gov/mmwr/preview/mmwrhtml/mm5745a3.htm.

5. CDC. [Nicotine Addiction: Past and Present] (CDC (US); National Center for Chronic Disease Prevention and Health Promotion (US); Office on Smoking and Health (US), 2010) (Date accessed: 10/03/2014). http://www.ncbi.nlm.nih.gov/ books/NBK53018/.

6. Kandel, D. B. \& Chen, K. Extent of smoking and nicotine dependence in the United States: 1991-1993. Nicotine Tob Res. 2, 263-274 (2000).

7. Slotkin, T. A. Nicotine and the adolescent brain: insights from an animal model. Neurotoxicol Teratol. 24, 369-384 (2002).

8. Benowitz, N. L. Pharmacologic Aspects of Cigarette Smoking and Nicotine Addiction. NEJM. 319, 1318-1330, doi:doi:10.1056/NEJM198811173192005 (1988).

9. Dani, J. A. \& Heinemann, S. Molecular and cellular aspects of nicotine abuse Neuron 16, 905-908 (1996).

10. Samaha, A. N., Yau, W. Y., Yang, P. \& Robinson, T. E. Rapid delivery of nicotine promotes behavioral sensitization and alters its neurobiological impact. Biol Psychiatry. 57, 351-360, doi:10.1016/j.biopsych.2004.11.040 (2005).

11. Wada, E. et al. Distribution of alpha 2, alpha 3, alpha 4, and beta 2 neuronal nicotinic receptor subunit mRNAs in the central nervous system: a hybridization histochemical study in the rat. J Comp Neurol. 284, 314-335, doi:10.1002/ cne.902840212 (1989).

12. Sobkowiak, R., Kowalski, M. \& Lesicki, A. Concentration- and time-dependent behavioral changes in Caenorhabditis elegans after exposure to nicotine. Pharmacol Biochem Behav. 99, 365-370, doi:10.1016/j.pbb.2011.05.019 (2011).

13. Feng, Z. et al. A C. elegans model of nicotine-dependent behavior: regulation by TRP-family channels. Cell 127, 621-633, doi:10.1016/j.cell.2006.09.035 (2006).

14. Thomas, L. T., Welsh, L., Galvez, F. \& Svoboda, K. R. Acute nicotine exposure and modulation of a spinal motor circuit in embryonic zebrafish. Toxicol Appl Pharmacol. 239, 1-12, doi:10.1016/j.taap.2008.08.023 (2009).

15. Counotte, D. S. et al. Long-lasting cognitive deficits resulting from adolescent nicotine exposure in rats. Neuropsychopharmacol. 34, 299-306, doi:10.1038/ npp.2008.96 (2009)

16. Ajarem, J. S. \& Ahmad, M. Prenatal nicotine exposure modifies behavior of mice through early development. Pharmacol Biochem Behav. 59, 313-318 (1998).

17. Holloway, A. C., Cuu, D. Q., Morrison, K. M., Gerstein, H. C. \& Tarnopolsky, $M$. A. Transgenerational effects of fetal and neonatal exposure to nicotine. Endocrine 31, 254-259 (2007).

18. El Marroun, H. et al. Prenatal Tobacco Exposure and Brain Morphology: A Prospective Study in Young Children. Neuropsychopharmacology 39, 792-800, doi:10.1038/npp.2013.273 (2014).

19. Maritz, G. S. Perinatal exposure to nicotine and implications for subsequent obstructive lung disease. Paediatr Respir Rev. 14, 3-8, doi:10.1016/ j.prrv.2012.03.006 (2013).

20. Taki, F., Pan, X. \& Zhang, B. Nicotine Exposure Caused Significant Transgenerational Heritable Behavioral Changes in Caenorhabditis elegans. EXCLI J. 12, 793-806 (2013).

21. Matta, S. G. et al. Guidelines on nicotine dose selection for in vivo research. Psychopharmacology 190, 269-319, doi:10.1007/s00213-006-0441-0 (2007).

22. Riddle, D. L., Blumenthal, T. \& B.J., M. [C. elegans II. 2nd edition.] (CSHL Press., 1997) (Date accessed: 10/11/2014) http://www.ncbi.nlm.nih.gov/books/ NBK20086/.

23. Brenner, S. The genetics of Caenorhabditis elegans. Genetics 77, 71-94 (1974).

24. Beitel, G. J., Clark, S. G. \& Horvitz, H. R. Caenorhabditis elegans ras gene let-60 acts as a switch in the pathway of vulval induction. Nature 348, 503-509, doi:10.1038/348503a0 (1990)

25. Lee, R. C., Feinbaum, R. L. \& Ambros, V. The C. elegans heterochronic gene lin-4 encodes small RNAs with antisense complementarity to lin-14. Cell 75, 843-854, doi:0092-8674(93)90529-Y (1993).

26. Zhang, B., Wang, Q. \& Pan, X. MicroRNAs and their regulatory roles in animals and plants. J Cell Physiol. 210, 279-289, doi:10.1002/jcp.20869 (2007).

27. Ambros, V. MicroRNA pathways in flies and worms: growth, death, fat, stress, and timing. Cell 113, 673-676, doi:S0092867403004288 (2003).

28. Aukerman, M. J. \& Sakai, H. Regulation of flowering time and floral organ identity by a MicroRNA and its APETALA2-like target genes. Plant Cell 15, 2730-2741, doi:10.1105/tpc.016238tpc.016238 (2003).

29. Chen, C. Z., Li, L., Lodish, H. F. \& Bartel, D. P. MicroRNAs modulate hematopoietic lineage differentiation. Science 303, 83-86, doi:10.1126/ science.10919031091903 (2004)

30. Kim, V. N. MicroRNA biogenesis: coordinated cropping and dicing. Nat Rev Mol Cell Biol 6, 376-385, doi:10.1038/nrm1644 (2005).

31. McManus, M. T. MicroRNAs and cancer. Semin Cancer Biol 13, 253-258 (2003).

32. Ng, T. K. et al. Nicotine Alters MicroRNA Expression and Hinders Human Adult Stem Cell Regenerative Potential. Stem Cells Dev. 22, 781-790, doi:10.1089/ scd.2012.0434 (2013)

33. Balaraman, S., Winzer-Serhan, U. H. \& Miranda, R. C. Opposing actions of ethanol and nicotine on microRNAs are mediated by nicotinic acetylcholine receptors in fetal cerebral cortical-derived neural progenitor cells. Alcohol Clin Exp Res. 36, 1669-1677, doi:10.1111/j.1530-0277.2012.01793.x (2012).

34. Kassie, F., Jarcho, M. \& Endalew, A. Abstract PR-06: Upregulation of microRNA21 (miR-21) in human bronchial epithelial cells chronically exposed to 4 
(methylnitrosamino)-1-(3-pyridyl)-1-butanone (NNK) plus nicotine and modulation of these effects by diindolylmethane. Cancer Prev Res 3, A51, doi:10.1158/1940-6207.PREV-09-PR-06 (2010).

35. Shan, H. et al. Downregulation of miR-133 and miR-590 contributes to nicotineinduced atrial remodelling in canines. Cardiovasc Res. 83, 465-472, doi:10.1093/ cvr/cvp130 (2009).

36. Huang, W. \& Li, M. D. Nicotine modulates expression of miR-140*, which targets the 3 '-untranslated region of dynamin 1 gene (Dnm1). Int $J$ Neuropsychopharmacol 12, 537-546, doi:10.1017/S1461145708009528 (2009).

37. Shin, V. Y. et al. NF-kappaB targets miR-16 and miR-21 in gastric cancer: involvement of prostaglandin E receptors. Carcinogenesis 32, 240-245, doi:10.1093/carcin/bgq240 (2011).

38. Zhang, B., Pan, X., Cobb, G. P. \& Anderson, T. A. microRNAs as oncogenes and tumor suppressors. Developmental Biology 302, 1-12, doi:http://dx.doi.org/10. 1016/j.ydbio.2006.08.028 (2007).

39. Sulston, J. \& Hodgkin, J. The Nematode Caenorhabditis elegans. 587. Wood, W. B. (ed.) (Cold Spring Harbor Laboratory Press, New York, (1988).

40. AI, S. et al. TM4 microarray software suite. Methods Enzymol. 411, 134-193 (2006).

41. Heikkinen, L., Kolehmainen, M. \& Wong, G. Prediction of microRNA targets in Caenorhabditis elegans using a self-organizing map. Bioinformatics 27 1247-1254, doi:10.1093/bioinformatics/btr144 (2011).

42. Huang, d. W., Sherman, B. \& Lempicki, R. Systematic and integrative analysis of large gene lists using DAVID Bioinformatics Resources. Nature Protoc 4, 44-57 (2009).

43. Huang, d. W., Sherman, B. \& Lempicki, R. Bioinformatics enrichment tools: paths toward the comprehensive functional analysis of large gene lists. Nucleic Acids Res 37, 1-13 (2009).

44. Eden, E., Navon, R., Steinfeld, I., Lipson, D. \& Yakhini, Z. GOrilla: a tool for discovery and visualization of enriched GO terms in ranked gene lists. BMC bioinformatics 10, 48, doi:10.1186/1471-2105-10-48 (2009).

45. Smoot, M. E., Ono, K., Ruscheinski, J., Wang, P. L. \& Ideker, T. Cytoscape 2.8: new features for data integration and network visualization. Bioinformatics 27, 431-432, doi:10.1093/bioinformatics/btq675 (2011).

46. Taki, F. A., Pan, X. \& Zhang, B. Chronic nicotine exposure systemically alters microRNA expression profiles during post-embryonic stages in Caenorhabditis elegans. J Cell Physiol., doi:10.1002/jcp.24419 (2014).

47. Yook, K. et al. WormBase 2012: more genomes, more data, new website. Nucleic Acids Res. 40, D735-D741, doi:10.1093/nar/gkr954 (2012)

48. Crews, D. et al. Epigenetic transgenerational inheritance of altered stress responses. PNAS. 109, 9143-9148, doi:10.1073/pnas.1118514109 (2012).

49. Skinner, M. K. What is an epigenetic transgenerational phenotype? F3 or F2. Reprod Toxicol 25, 2-6, doi:10.1016/j.reprotox.2007.09.001 (2008).

50. Skjei, K. L. \& Markou, A. Effects of repeated withdrawal episodes, nicotine dose and duration of nicotine exposure on the severity and duration of nicotine withdrawal in rats. Psychopharmacology 168, 280-292, doi:10.1007/s00213-0031414-1 (2003).

51. Furuhashi, H. et al. Trans-generational epigenetic regulation of C. elegans primordial germ cells. Epigenetics \& chromatin 3, 15, doi:10.1186/1756-8935-3-15 (2010).

52. Kolokotroni, K. Z., Rodgers, R. J. \& Harrison, A. A. Effects of chronic nicotine nicotine withdrawal and subsequent nicotine challenges on behavioural inhibition in rats. Psychopharmacology 219, 453-468, doi:10.1007/s00213-0112558-z (2012).
53. Lim, L. P. et al. The microRNAs of Caenorhabditis elegans. Genes Dev 17, 991-1008, doi:10.1101/gad.1074403 (2003).

54. Brenner, J. L., Jasiewicz, K. L., Fahley, A. F., Kemp, B. J. \& Abbott, A. L. Loss of individual microRNAs causes mutant phenotypes in sensitized genetic backgrounds in C. elegans. Current biology: CB 20, 1321-1325, doi:10.1016/ j.cub.2010.05.062 (2010).

55. Kato, M., de Lencastre, A., Pincus, Z. \& Slack, F. J. Dynamic expression of small non-coding RNAs, including novel microRNAs and piRNAs/21U-RNAs, during Caenorhabditis elegans development. Genome Biol 10, R54, doi:10.1186/gb-200910-5-r54 (2009).

56. Lau, N. C., Lim, L. P., Weinstein, E. G. \& Bartel, D. P. An abundant class of tiny RNAs with probable regulatory roles in Caenorhabditis elegans. Science 294, 858-862, doi:10.1126/science.1065062 (2001).

57. McPherson, C. S. \& Lawrence, A. J. The nuclear transcription factor CREB: involvement in addiction, deletion models and looking forward. Curr Neuropharmacol. 5, 202-212, doi:10.2174/157015907781695937 (2007).

58. Larson, E. B. et al. Overexpression of CREB in the nucleus accumbens shell increases cocaine reinforcement in self-administering rats. J Neurosci. $\mathbf{3 1}$, 16447-16457, doi:10.1523/JNEUROSCI.3070-11.2011 (2011).

59. Vonhoff, F., Kuehn, C., Blumenstock, S., Sanyal, S. \& Duch, C. Temporal coherency between receptor expression, neural activity and AP-1-dependent transcription regulates Drosophila motoneuron dendrite development. Development 140, 606-616, doi:10.1242/dev.089235 (2013).

60. Maze, I. \& Nestler, E. J. The epigenetic landscape of addiction. Ann N Y Acad Sci. 1216, 99-113, doi:10.1111/j.1749-6632.2010.05893.x (2011).

\section{Acknowledgments}

The project is partially supported by Grant Number R03DA032515 from the National Institute on Drug Abuse. The content is solely the responsibility of the authors and does not necessarily represent the official views of the National Institute on Drug Abuse or the National Institutes of Health.

\section{Author contributions}

F.T., X.P., M.H.L. and B.Z. designed the experiments, analyzed the data and wrote the main manuscript text. F.T. performed the experiments. All authors reviewed the manuscript.

\section{Additional information}

Competing financial interests: The authors declare no competing financial interests.

How to cite this article: Taki, F.A., Pan, X., Lee, M.-H. \& Zhang, B. Nicotine exposure and transgenerational impact: a prospective study on small regulatory microRNAs. Sci. Rep. 4, 7513; DOI:10.1038/srep07513 (2014).

This work is licensed under a Creative Commons Attribution-NonCommercialShareAlike 4.0 International License. The images or other third party material in this article are included in the article's Creative Commons license, unless indicated otherwise in the credit line; if the material is not included under the Creative Commons license, users will need to obtain permission from the license holder in order to reproduce the material. To view a copy of this license, visit http:// creativecommons.org/licenses/by-nc-sa/4.0/ 